\title{
Glycerol kinase-like proteins cooperate with PId6 in regulating sperm mitochondrial sheath formation and male fertility
}

\author{
Yuxi Chen ${ }^{1,2,3,6}$, Puping Liang ${ }^{1,2,3,6}$, Yan Huang ${ }^{1,3,6}$, Minyan $\mathrm{Li}^{1,3}$, Xiya Zhang ${ }^{1,3}$, Chenhui Ding ${ }^{3}$, \\ Junyan Feng, ${ }^{1,3}$, Zhen Zhang ${ }^{1,3}$, Xueqing Zhang ${ }^{1}$, Yuanzhu Gao ${ }^{1}$, Qinfeng Zhang ${ }^{1}$, Shanbo Cao ${ }^{4}$, \\ Haiyan Zheng ${ }^{4}$, Dan Liu ${ }^{5}$, Zhou Songyang ${ }^{1,2,3,5, *}$ Junjiu Huang ${ }^{1,2,3,4, *}$ \\ ${ }^{1}$ Key Laboratory of Gene Engineering of the Ministry of Education, Institute of Healthy Aging Research and State Key \\ Laboratory of Biocontrol, SYSU-BCM Joint Research Center, School of Life Sciences, Sun Yat-sen University, Guangzhou, \\ China; ${ }^{2}$ State Key Laboratory of Ophthalmology, Zhongshan Ophthalmic Center, Sun Yat-sen University, Guangzhou, China; \\ ${ }^{3}$ Key Laboratory of Reproductive Medicine of Guangdong Province, School of Life Sciences and the First Affiliated Hospital, Sun \\ Yat-sen University, Guangzhou, China; ${ }^{4}$ Key Laboratory of Reproductive Medicine of Guangdong Province, Third Affiliated \\ Hospital of Guangzhou Medical University, Guangzhou, China, ${ }^{5}$ Cell-Based Assay Screening Core, Verna and Marrs Mclean \\ Department of Biochemistry and Molecular Biology, Baylor College of Medicine, One Baylor Plaza, Houston, TX, USA
}

\begin{abstract}
Spermatids undergo the final steps of maturation during spermiogenesis, a process that necessitates extensive rearrangement of organelles such as the mitochondria. Male infertility has been linked to mitochondrial disorder, for example, hypospermatogenesis and asthenozoospermia. However, the mechanisms that regulate mitochondrial dynamics during spermiogenesis remain largely unknown. We found the glycerol kinase (Gyk)-like proteins glycerol kinase-like 1 (Gykl1) and glycerol kinase 2 (Gk2) were specifically localized to the mitochondria in spermatids. Male mice deficient in either Gykl1 or $G k 2$ were infertile due to dysfunctional spermatozoa, which exhibited unregulated ATP production, disordered mitochondrial sheath formation, abnormal mitochondrial morphology, and defective sperm tail. We demonstrated that the unique C-terminal sequences found in Gykl1 and Gk2 mediated their targeting to the mitochondrial outer membrane. Furthermore, both Gykl1 and Gk2 could interact with Pld6 (MitoPLD) and induce PId6 and phosphatidic acid (PA)dependent mitochondrial clustering in cells. Taken together, our study has revealed previously unsuspected functions of Gyk-like proteins in spermiogenesis, providing new insight into the potential mechanisms that lead to spermatozoa dysfunction and male infertility.
\end{abstract}

Cell Discovery (2017) 3, 17030; doi:10.1038/celldisc.2017.30; published online 22 August 2017

\section{Introduction}

Mitochondria are sites of oxidative phosphorylation and essential for cellular energy production [1]. Mitochondria are highly dynamic and motile. They change in shape and size and undergo fusion, fission, transport and mitophagy, which involve complicated steps and

\footnotetext{
${ }^{6}$ These three authors contributed equally to this work.

*Correspondence: Zhou Songyang

Tel: +020 39943531 ;

E-mail: songyang@bcm.edu

or Junjiu Huang

Tel: 020-39943531; Fax: 020-39943778

E-mail: hjunjiu@mail.sysu.edu.cn

Received 24 April 2017; accepted 23 July 2017
}

require coordination [2-4]. For example, arrangement of mitochondria may depend on their trafficking along the cytoskeleton [5-7]. In response to internal and external stimuli, mitochondria undergo constant fusion and fission. Fusion may occur to ensure mitochondrial homogeneity in response to metabolic and environmental stress, and fission can eliminate damaged mitochondria and facilitate apoptosis [2].

While the exact mechanisms and physiological significance of mitochondria dynamics remain under active investigation, multiple players have been uncovered. For instance, both mitochondrial fusion and fission appear to be mediated by mitochondrial membrane proteins and dynamin family members 
[4, 8-13]. One widely studied regulatory pathway involves lipids such as cardiolipid (CL), phosphatidic acid (PA), diacylglycerol (DAG) and lysophosphatidic acid (LPA) $[14,15]$. The observation that phospholipase D family member 6 (Pld6/MitoPLD) induces mitochondrial fusion by generating PA from CL indicates that PA may act as a signaling molecule in controlling mitochondrial dynamics [4]. Further investigations showed that the phosphatidate phosphatase LIPIN1 could terminate mitochondrial fusion by converting PA into DAG [16]. Phosphatidic acidpreferring phospholipase A1 (Pa-pla 1$)$ can also deacylate PA into LPA, thus inhibiting mitochondrial fusion induced by Pld6 [1].

Normal mitochondrial function and dynamics are intimately linked to biological processes such as cell growth, proliferation and differentiation. For instance, the number, shape, structure and dynamics of mitochondria change dramatically during mammalian spermatogenesis. Secondary spermatocytes, spermatids, and sperms have more condensed forms of mitochondria compared with spermatogonia and primary spermatocytes [17]. During spermiogenesis, the mitochondria undergo reduction and rearrangement and form tubular structures that make up the sperm midpiece $[18,19]$. The mitochondrial sheath is tightly packed in the midpiece of the sperm flagellum and is thought to be responsible for generating the ATP required for sperm motility $[7,20]$. The evolutionarily conserved mitochondrial sheath structure, which is important for spermatozoa motility, underlines the distinct and important function of mitochondria in spermatogenesis [17, 19, 21]. A number of proteins have been reported to be involved in mitochondrial dynamics during spermatogenesis, including the PTEN-induced putative kinase 1 (PINK1), Pld6 and Pa-pla $_{1}[1,4,8,16,22]$. Male infertility has been linked to mitochondrial disorders such as hypospermatogenesis with abnormal lower mtHSP60 immunoreactivity and asthenozoospermia with mutation of the $\mathrm{CO}_{\mathrm{III}}$ gene $[23,24]$. Collectively, these studies demonstrate that tight regulation of mitochondrial function is essential for proper spermatogenesis. However, the regulatory process that modulates the mitochondrial sheath formation during spermiogenesis remains to be identified.

We report here the identification of glycerol kinase (Gyk)-like proteins glycerol kinase-like 1 (Gykl1) and glycerol kinase $2(\mathrm{Gk} 2)$ as new players that specifically regulate sperm mitochondrial sheath formation. Gyk is the enzyme responsible for converting glycerol into glycerol 3-phosphate, the substrate for glycolysis and lipid synthesis [25-28]. Two Gyk-related genes Gykll and $G k 2$ were first identified as Gyk-like intronless retroposons and found exclusively expressed in mouse testis [29, 30]. Mouse Gyk11 (549 a.a.) and Gk2 (554 a. a.) are highly similar in protein sequence but differ slightly in molecular weight. Human appears to have only one glycerol kinase (Gyk)-like protein, GK2 [29]. In contrast to Gyk, neither Gyk11 nor Gk2 exhibits detectable glycerol kinase activity in vitro [30]. Interestingly, decreased GK2 mRNA expression in males with teratozoospermia and increased GK2 protein levels in asthenozoospermia patients have also been reported [31, 32], suggesting regulated expression of GK2 may be a key factor in male fertility.

We found that Gyk11 and Gk2 were exclusively expressed and localized to mitochondria in round and elongated spermatids and retained on the mitochondrial sheath in spermatozoa. Furthermore, deletion of either Gykll or Gk2 in mice, by CRISPR/Cas9-mediated gene knockout (KO), impacted mitochondrial morphology and mitochondrial ATP production as well as midpiece and sperm tail integrity in spermatozoa, compromising spermatozoa motility and leading to male infertility. Gyk11 or Gk2 may anchor itself to the mitochondrial outer membrane through its C-terminal mitochondrial localization sequence. Overexpression of Gykl1 or Gk2-induced mitochondrial clustering in a PA-dependent manner. Both Gykl1 and Gk2 can interact with Pld6, possibly serving as activators of Pld6 function. Our data not only shed light on the factors and pathways that regulate mitochondrial dynamics and integrity but also underscore the crucial role played by Gyk-like proteins during spermiogenesis. These findings also have important clinical implications for patients with mitochondriarelated reproductive disorders, and for the development of new and effective contraceptives.

\section{Results}

\section{Mouse Gykl1 and Gk2 are specifically expressed in round and elongating spermatids}

In a search for specific spermatogenesis-regulatory proteins, we identified mouse Gyk-like proteins Gykl1 and Gk2 as two possible candidates [33]. Gyk is highly conserved across species. Patients with X-linked recessive glycerol kinase deficiency (GKD), which is caused by enzymatic inactivation or Gyk deletion [34], suffer from asymptomatic hyperglyceridaemia, growth and psychomotor retardation and metabolic disorders $[25,34,35]$. Knockout of $G y k$ in mice leads to postnatal growth retardation, abnormal fat metabolism, 


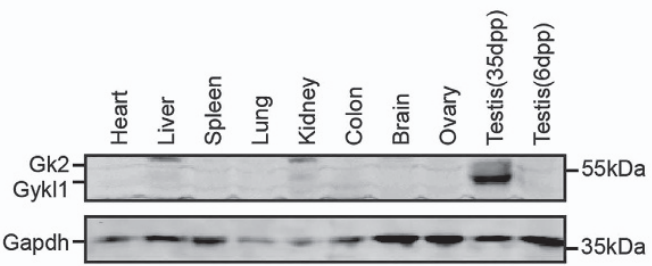
WT ACCAGCGGGAAAC TACGA TA-GTCTGGG

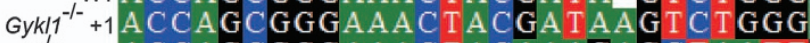
$6 y k 11^{-1-}+236$ ACCAGCGGGAAACTACAAAGAGCTAAT T gRNA 753

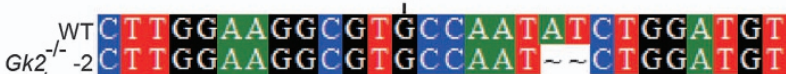
$\mathrm{Gk}^{-/-}-14 \mathrm{C} \mathrm{T} \mathrm{T} \mathrm{GGAAGGCGT} \mathrm{G \sim \sim \sim \sim \sim \sim \sim \sim \sim \sim \sim \sim \sim \sim \textrm {T }}$

e

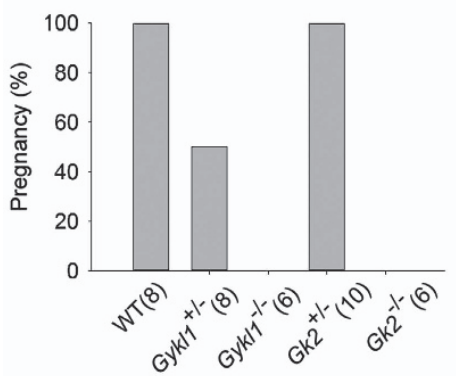

f

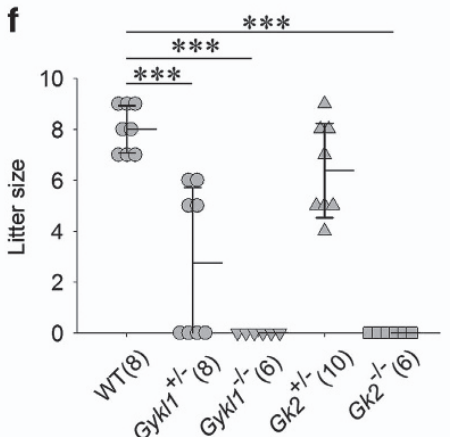

Gykl1/Gk2

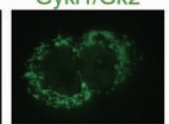

MitoTracker

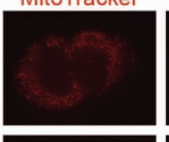

Merge

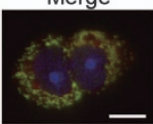

.
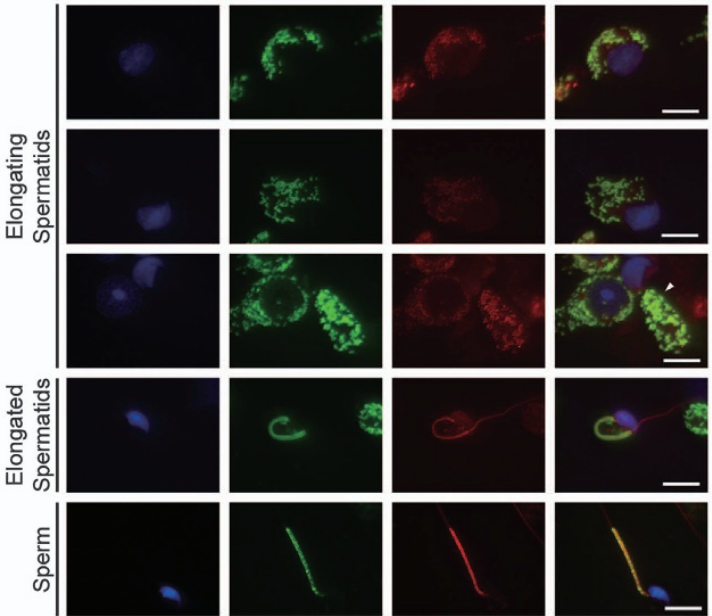

d
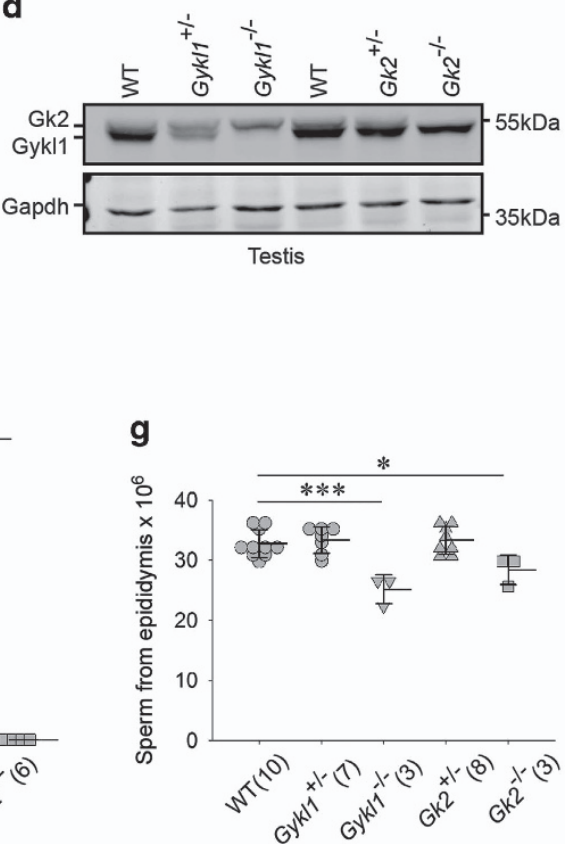

Figure 1 Mice deficient in Gykl1 or Gk2 exhibit reproductive defects. (a) Immunoblotting of Gykl1 and Gk2 protein in various tissues from C57BL/6J mice. dpp: day post partum. (b) Immunostaining of Gykl1 and Gk2 in round spermatids, elongating spermatids and spermatozoa pre-stained with $150 \mathrm{nM}$ MitoTracker Red CMXRos. White arrow indicates the mitochondria in elongating spermatid. Scale bar: $10 \mu \mathrm{m}$. (c) Gykl1 and Gk2 KO mice were generated by CRISPR/Cas9-mediated genome editing. sgRNA sequences that target the Gykl1 and Gk2 loci are indicated here. Wild-type (WT) sequences are aligned against sequences from both mutant alleles for each mutant mouse. ' $n$ ' denotes the number of nucleotides inserted or deleted in the region. (d) Whole-cell extracts were collected from the testes of wild-type and Gyk/1/Gk2 KO mice, and blotted with antibodies against Gykl1/Gk2. An antibody against GAPDH was used as a loading control. (e- $\mathbf{g})$ The mean pregnancy rate (e), litter size (f) and sperm count (from the epididymis) (g) for wild-type vs homozygous (-/ - ) and heterozygous (+/-) Gyk/1 and Gk2-deficient mice were determined and compared. Error bars represent s.d. Numbers in parentheses indicate sample size $(n)$. Significance was determined by ANOVA. ${ }^{*} P<0.05$. ${ }^{* \star} P<0.01$. ${ }^{* * *} P<0.001$. 
autonomous glucocorticoid synthesis, and early death (within 3-4 days) [25]. Gyk11 and Gk2 are highly similar to Gyk and to each other (Supplementary Figure S1A). However, they exhibit no glycerol kinase activities in vitro and their functions remain unknown [30]. Unlike $G y k$, which was detectable in the kidney and brain of C57BL/6J mice, Gykll and Gk2 mRNAs were specifically found in mouse testis (Supplementary Figure S1B) [29, 33]. To examine the protein expression pattern of Gyk11 and Gk2 in different tissues, we raised a rabbit polyclonal antibody against recombinant full-length Gyk11 protein. The antibody could detect ectopically expressed Gyk11 and Gk2 in HEK293T cells (Supplementary Figure S1C), as well as endogenous Gyk11 and Gk2 proteins in mouse testes (Figure 1a), and henceforth was designated anti-Gyk11/Gk2 antibody. Consistent with their mRNA expression patterns, Gyk11 and Gk2 protein expression appeared to be restricted to mature testes (Figure 1a).

The first wave of mouse spermatozoa appears between 1 and 35 days post partum (dpp), representing the developmental stages of spermatogenesis. During this process, spermatogonia (1-6 dpp) differentiate into primary and secondary spermatocytes (7-14 dpp) and later into round (15-21 dpp) and elongating spermatids (22-35 dpp) [36]. When we examined the expression of Gyk11/Gk2 in lysates of testes from different days post partum, we found strong signals of Gyk11 and Gk2 in 35, 49 and $70 \mathrm{dpp}$ samples, indicative of their expression in spermatids (Supplementary Figure S1D). Immunostaining of seminiferous tubules at different spermatogenesis stages showed intense expression of Gykl1 and Gk2 protein at 21 and $35 \mathrm{dpp}$, indicating the expression of Gykl1 and Gk2 is as early in round spermatids (Supplementary Figure S1E). To further investigate the specific cell type and developmental stages in which Gyk11 and Gk2 were expressed, testis sections from $35 \mathrm{dpp}$ C57BL/6J mice were stained with the Gyk11/Gk2 antibody along with antibodies against the spermatogonia stem cell marker PLZF and the meiosis marker SYCP3 (Supplementary Figure S1F). Only cells close to the lumen stained positive for Gyk11/Gk2. No overlap of signals was found for Gyk11/Gk2 with either PLZF or SYCP3, supporting the notion that Gyk11 and Gk2 proteins were restricted to round and elongating spermatids and absent from spermatogonia and spermatocytes. We speculated that the punctate and tubular staining patterns of Gyk11 and Gk2 in round and elongating spermatids were suggestive of mitochondrial localization, and proceeded to co-stain round and elongating spermatids from testis and mature spermatozoa from epididymis for Gyk11/Gk2 and MitoTracker. Indeed, we found overlapping signals of Gyk11/Gk2 with MitoTracker (Figure 1b), strongly suggesting that Gykl1 and $\mathrm{Gk} 2$ are specifically localized to the mitochondria in round and elongating spermatids.

\section{Male mice deficient in Gykll or Gk2 display reproductive defects}

To better understand the function of Gykll and Gk2 in vivo, we generated mice knocked out for either Gykll or Gk2 using CRISPR/Cas9 as previously described, and obtained two mutant lines for each gene (Figure 1c) [37-39]. Genomic sequencing revealed frame-shift mutations that resulted in premature translation termination in all cases (Figure 1c). Successful disruption of Gyk11 or Gk2 expression was confirmed by Western blotting of protein extracts of the testis using our anti-Gykl1/Gk2 antibody (Figure 1d). Deletion of Gykll did not appear to affect the expression of $G k 2$ and vice versa (Figure 1d). We found no gross changes compared with controls in testis morphology and size or germ cell arrangements in the seminiferous tubules in the $\mathrm{KO}$ mice (Supplementary Figure S2A-S2C), suggesting normal testis morphogenesis in the mutant mice.

When mated with wild-type C57BL/6J females, $G y k l 1^{-1-}$ and $G k 2^{-/-}$males appeared infertile, and significant reduction $(50 \%)$ in pregnancy rates was noted for the $\mathrm{GyklI}^{+-}$mice (Figure 1e). The litter size of $\mathrm{GyklI}^{+-}$ males $(2.8 \pm 3.0)$ was significantly smaller than that of WT mice $(8.0 \pm 1.0)$ (Figure 1f). Furthermore, fewer spermatozoa were obtained from the epididymis of $\mathrm{Gykll}^{-/-}$and $G k 2^{-1-}$ mice $\left(3.28 \times 10^{7}\right.$ for WT, $2.52 \times 10^{7}$ for $G_{y k l 1^{-1-}}$, and $2.84 \times 10^{7}$ for $G k 2^{-/-}$) (Figure $1 \mathrm{~g}$ ). Therefore, $G y k l 1^{-1-}$ and $G k 2^{-1-}$ mice clearly display reproductive defects despite apparently normal testis morphogenesis.

\section{Gykl1 ${ }^{-1-}$ and $\mathrm{Gk}^{-{ }_{-1}}$ males exhibit mitochondrial defects in the spermatozoa}

To probe the causes of infertility and reproductive defects in Gykll and Gk2 KO mice, we carried out computer-assisted spermatozoa analysis (CASA) to document spermatozoa motility, including curvilinear velocity, straight-line velocity, and amplitude of lateral head movement in the mutant mice. As shown by all the parameters we examined (Table 1; Supplementary Movies S1 and S2), spermatozoa deficient for Gykll or Gk2 showed drastically reduced activities.

Under microscopes, normal spermatozoa appear relaxed or bent (resembling a smooth string), with the midpiece connected smoothly to the tail (Figure 2a). In Gykll-KO spermatozoa, however, a gap between the 
Table 1 Impact of Gykl1 or Gk2 knockout on spermatozoa mitility

\begin{tabular}{|c|c|c|c|c|c|}
\hline Parameter & $W T$ & Gykl1 ${ }^{+/-}$ & Gykl1 ${ }^{-1-}$ & $G k 2^{+/-}$ & $G k 2^{-l-}$ \\
\hline Motile (\%) & $52.75 \pm 8.6$ & $39.82 \pm 7.57$ & $26.05 \pm 4.84^{* * *}$ & $50.91 \pm 12.79$ & $21.12 \pm 6.27^{* * *}$ \\
\hline CurviLinear velocity $\left(\mu \mathrm{m} \mathrm{s}^{-1}\right)$ & $200.41 \pm 19.79$ & $156.59 \pm 9.27^{* *}$ & $132.19 \pm 20.06^{* *}$ & $174.87 \pm 42.55$ & $106.91 \pm 16.6^{* * *}$ \\
\hline Straight-line velocity $\left(\mu \mathrm{m}^{-1}\right)$ & $62.51 \pm 6.66$ & $27.75 \pm 8.87^{* * *}$ & $22.67 \pm 9.33^{* * *}$ & $50.5 \pm 10.97 *$ & $24.54 \pm 10.51^{* * *}$ \\
\hline Average path velocity $\left(\mu \mathrm{m} \mathrm{s}^{-1}\right)$ & $95.59 \pm 7.56$ & $56.48 \pm 12.05^{* * *}$ & $46.24 \pm 10.94^{* * *}$ & $81.08 \pm 19.12$ & $43.69 \pm 13.45^{* * *}$ \\
\hline Amplitude of lateral head movement $(\mu \mathrm{m})$ & $11.37 \pm 1.17$ & $6.62 \pm 0.81 *$ & $4.53 \pm 2.55^{*}$ & $8.37 \pm 2.47$ & $4.48 \pm 2.67 *$ \\
\hline Beat-cross frequency $(\mathrm{Hz})$ & $6.12 \pm 0.41$ & $4.04 \pm 1.39 *$ & $2.42 \pm 1.38^{* *}$ & $6.71 \pm 0.73$ & $1.97 \pm 1.95^{* *}$ \\
\hline
\end{tabular}

Abbreviations: GyK, glycerol kinase; WT, wild type. Parameters of spermatozoa were analyzed by Computer-Assisted Spermatozoa Analysis system. Data are mean \pm s.d., $n=3-7,{ }^{*} P<0.05,{ }^{* *} P<0.01,{ }^{* * *} P<0.001$.

midpiece and tail was clearly present due to an abrupt bending that affected its morphology. The same abnormal hairpin-shaped morphology was also found in spermatozoa from $\mathrm{Gykll}^{+-}$mice. Interestingly, the midpiece of the spermatozoa from $G k 2^{-1-}$ mice was split into several pieces (Figure 2a). The percentage of abnormally shaped spermatozoa was significantly high in mice carrying homozygous or heterozygous deficient for Gykll $\left(^{+-}: 93.1 \pm 0.9 \%,{ }^{-1-}: 96.3 \pm 1.6 \%\right)$ or $G k 2$ $\left(^{+--}: 13.9 \pm 5.2 \%,^{-1-}: 96.9 \pm 1.2 \%\right.$ ) (Figure 2b). Moreover, the spermatozoa from $G y k l 1^{-1-}$ mice swam with their heads facing backward and twisting tails, a sign of severely impaired spermatozoa motility (Supplementary Movie S1).

Staining of the mitochondrial sheath in the affected spermatozoa revealed obvious abnormalities in the mitochondria. A gap was apparent between the midpiece and tail in the spermatozoa from heterozygous Gykll-KO mice (Figure 2c). An even larger gap was observed in Gykll deficient spermatozoa, with intense mitochondria staining (with the MitoTracker dye) at the end of the mitochondrial sheath. The spermatozoa tail was easily bent at these sites (Figure 2c). The length of the mitochondrial sheath was significantly shorter in the spermatozoa from Gykll- or Gk2-deficient mice (For example, $15.25 \pm 2.89 \mu \mathrm{m}$ for $\mathrm{Gykl1}^{-1-}$ vs $21.28 \pm 1.41 \mu \mathrm{m}$ for WT) (Figure $2 \mathrm{~d}$ and e). Additionally, intracytoplasmic spermatozoa injection (ICSI) of Gykll- or Gk2-KO spermatozoa led to normal offspring (Supplementary Table S1), suggesting that their infertility may be accounted to defects in fertilization. These results suggest that reduced spermatozoa motility induced by the disrupted mitochondrial sheath is the likely culprit for the infertility of Gykll- or Gk2-deficient mice.

\section{Abnormal mitochondrial sheath and sperm tail fragility are found in spermatozoa from Gykl1 - and Gk2-deficient mice}

Given the possible defects in the mitochondria sheath in the spermatozoa from Gykl1/Gk2 KO mice, we decided to examine these spermatozoa more carefully using transmission electron microscopy (TEM) coupled with Electron Tomography, which enables more detailed and 3D imaging. As expected, in wildtype (WT) spermatozoa, the mitochondria were neatly lined up along the central axoneme (Figure 3a). However, arrangement of the mitochondria was considerably disordered in Gykll-KO spermatozoa, indicating dysregulated mitochondrial sheath formation (Figure $3 \mathrm{~b}$ and $\mathrm{c}$, white arrows). Moreover, these mitochondria were of all shapes and sizes. In $G k 2-\mathrm{KO}$ spermatozoa, the mitochondria were relatively uniform in morphology and size, but they were missing in some areas along the mitochondria sheath, leaving obvious gaps (Figure 3d). The mitochondria in wild-type spermatozoa had a diameter of $\sim 100 \mathrm{~nm}$ in their longitudinal section, representing the length of the short axis (Figure $3 \mathrm{a}$ and e). In comparison, the mitochondria in Gykll-and Gk2-deficient spermatozoa looked large and swollen, nearly doubling the length of the short axis $(\sim 180 \mathrm{~nm})$ of wild-type mitochondria (Figure 3e).

The gaps observed under light microscopy (Figure 2a) were clearly visible with TEM (Figure 3a). Compared with wild-type spermatozoa, large gaps between the mitochondrial sheath and outer dense fibers appeared in both Gykll and Gk2 KO spermatozoa (Figure 3b-d, red arrows). In these regions, the mitochondrial sheath or the outer dense fibers were completely absent, leaving the axoneme 'unprotected'. Moreover, the outer dense fibers had larger gaps in the KO samples (WT: $30.51 \pm 11.63 \mathrm{~nm}, \quad$ Gykl1 ${ }^{-1-}$ : $\left.42.77 \pm 18.10 \mathrm{~nm}, G k 2^{-1-}: 63.84 \pm 26.72 \mathrm{~nm}\right)$, taking on a 'beaded string' look with enlarged gaps within them (Figure 3b-d, green arrows, and Figure 3f). Sperm tail integrity was greatly compromised in the spermatozoa of Gykl1- and Gk2-deficient mice, with evidence of breaking (Figure 3b-d, yellow arrows). These defects likely affected the activity of the spermatozoa (Table 1), resulting in reproductive abnormalities 
a
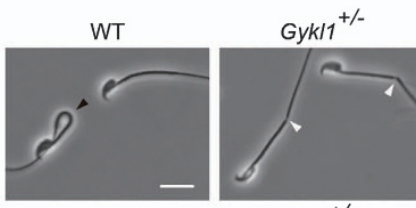

$G k 2^{+/-}$

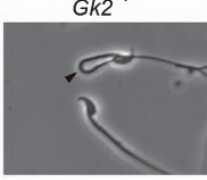

c

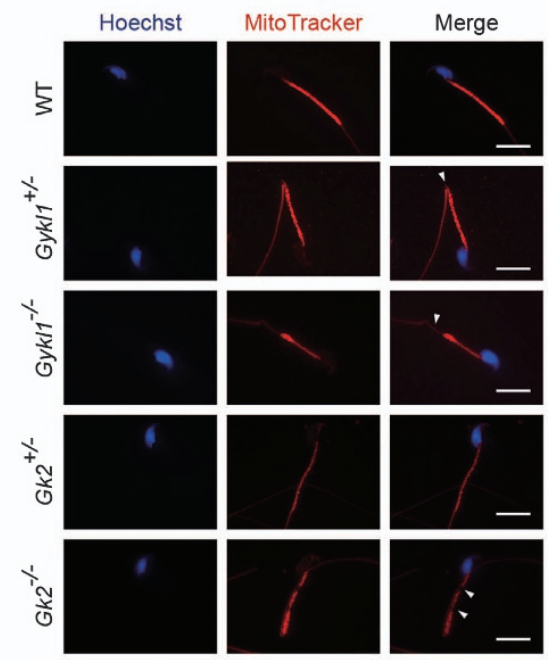

b

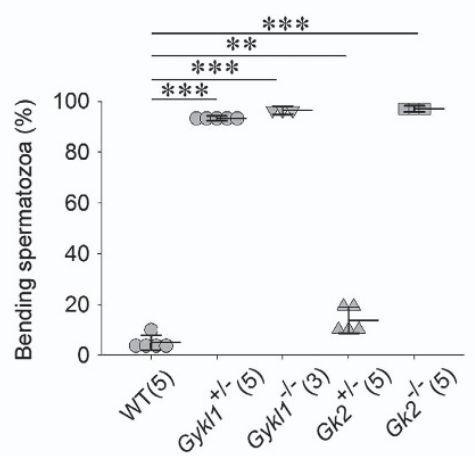

e
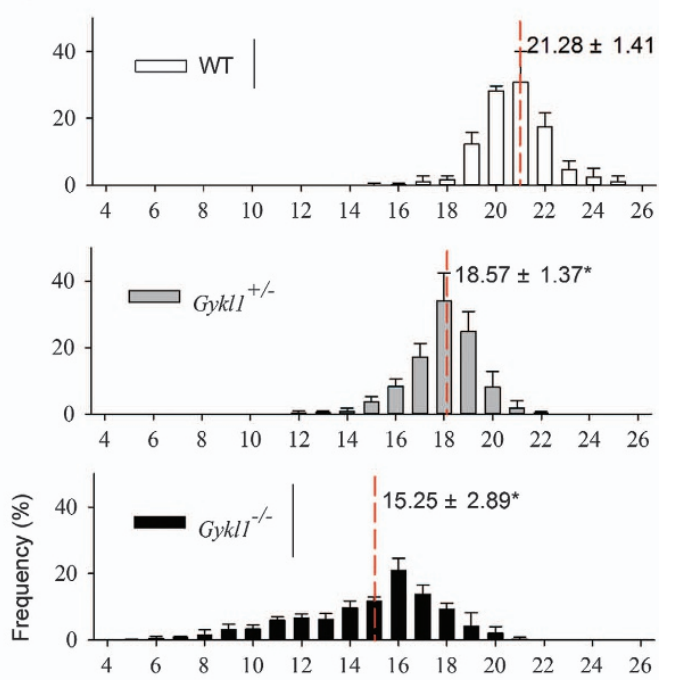

d
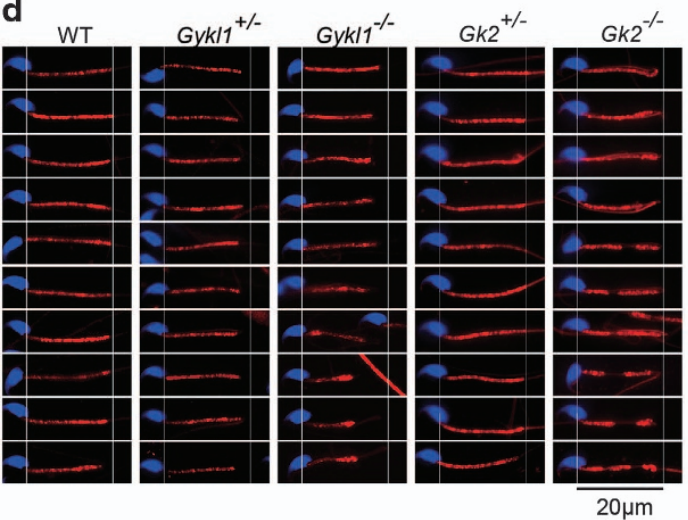
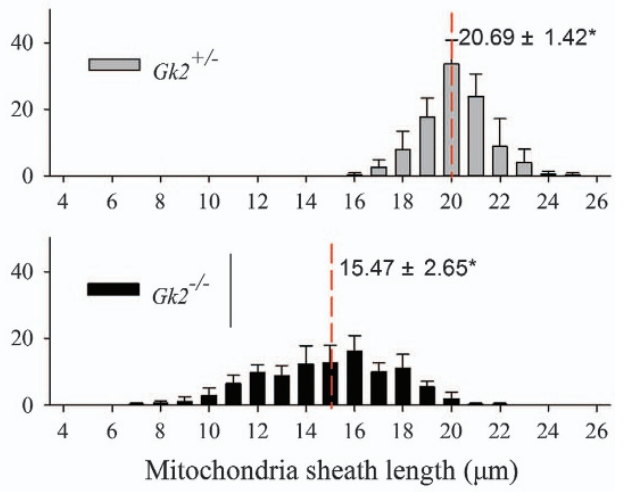

Figure 2 The spermatozoa from male Gykl1 and Gk2 KO mice display mitochondrial defects. (a) The spermatozoa from the epididymis of wild-type and mutant mice were visualized under the microscope. Black arrows indicate normal bending of sperms which is smooth. White arrows indicate abnormal gaps between the mitochondrial sheath and tail. (b) The spermatozoa from (a) were scored and plotted to calculate the mean percentage of spermatozoa with bending (e.g., hairpins or gaps). Error bars indicate s.d. Numbers in parentheses indicate sample size $(n)$. Significance was determined by the ANOVA. ${ }^{* *} P<0.01$. ${ }^{* \star *} P<0.001$. (c) The spermatozoa from various mice were stained with MitoTracker dye to visualize the mitochondria and Hoechst 33342 was used to visualize the nuclei. White arrows indicate gaps between the mitochondrial sheath and tail. Scale bar: $10 \mu \mathrm{m}$. (d and $\mathbf{e}$ ) The spermatozoa from various mice were collected to measure the length of mitochondrial sheath. Some representative spermatids measured in $\mathbf{e}$ are shown in $\mathbf{d}$. The distribution of the measurements is plotted in $\mathbf{e}$. The red line indicates average length. Error bars represent s.d. More than 300 spermatozoa were collected from 3-5 animals of each group. Significance was determined by the ANOVA. ${ }^{*} P<0.05$, compared with WT. 
a

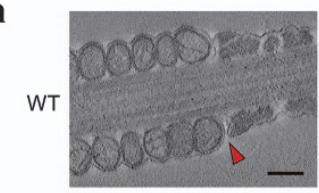

b

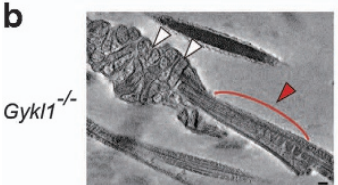

c

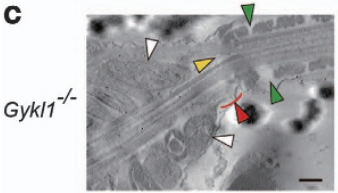

d

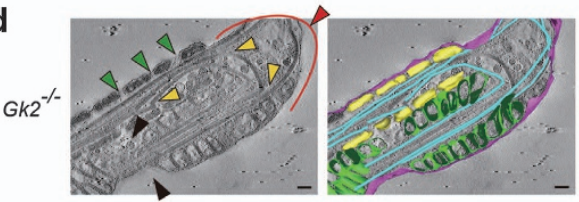

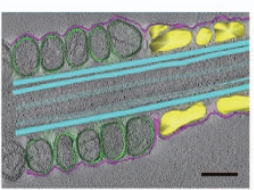
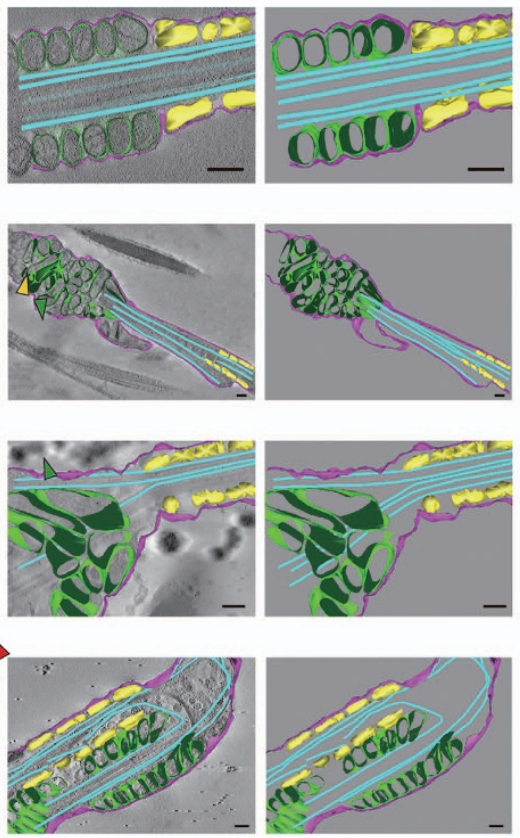

e

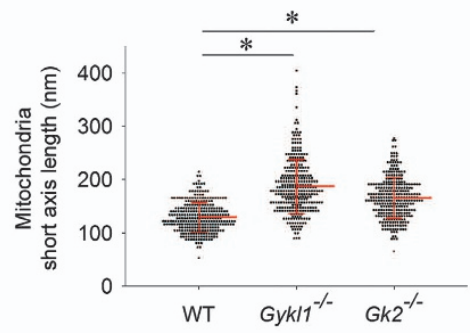

$\mathbf{f}$

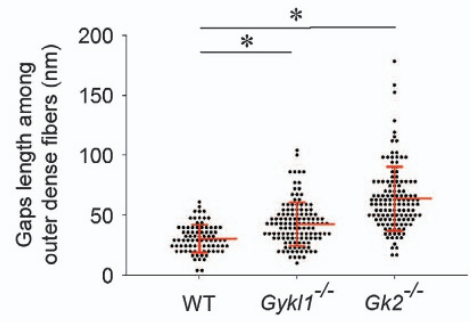

Figure 3 Ultra-structural analysis of the spermatozoa from male Gykl1 and Gk2 mutant mice. (a-d) The spermatozoa were collected from various mice and analyzed by transmission electron microscopy (TEM) coupled with Electron Tomography, which enabled detailed 3D imaging of the midpiece and tail regions. For each group, the middle slice of the electron tomogram is shown on the left, the annotation model on the right, and superimposed tomogram with the annotation model in the middle. Purple indicates the outer cell membrane of the spermatozoa, green the outer membrane of the mitochondria, cyan the doublet microtubule or central microtubule, and yellow the outer dense fibers. White arrows, swollen and misarranged mitochondria. Black arrows, midpiece that lacks mitochondria. Green arrows, gaps between outer dense fibers. Red arrows, gaps between the mitochondrial sheath and outer dense fibers. Yellow arrows, branched or fragile axoneme. Scale bar, $200 \mathrm{~nm}$. (e and f) The length of the mitochondria short axis (e) and the gaps among outer dense fibers (f) in the spermatozoa from wild-type and mutant mice were measured and plotted. Over 300 spermatozoa were counted for each group. Error bars represent s.d. Significance was determined by ANOVA. ${ }^{*} P<0.05$.

(Figure 1d and e). Regarding the observation of decreased GK2 in teratozoospermia [31], the Gykll or Gk2 KO mice did show abnormalities in morphology defected spermatozoa, which again raised the possible link between male infertility and Gyk-like proteins. Our analysis thus far indicates that Gyk11 and Gk2 may regulate mitochondrial arrangement and morphology, and the maintenance of sperm tail integrity in spermatozoa.

\section{Gykl1 and Gk2 contain specific sequences for mitochondrial targeting}

Our anti-Gyk11/Gk2 antibody recognizes both Gyk11 and Gk2 (Figure 1; Supplementary Figure S1), and therefore cannot reveal any possible differences in mitochondrial localization between the two proteins. To better understand the localization and function of Gyk11 vs Gk2, we GFP-tagged Gyk, Gyk11 and Gk2 at the $\mathrm{N}$ terminus and transiently expressed these proteins in HEK293T cells. As expected, GFP-Gyk exhibited primarily diffused cytoplasmic staining with no apparent mitochondrial localization, which was further confirmed by fractionation experiments (Figure $4 \mathrm{a}$ and b). In support of our data with endogenous Gyk11 and Gk2 (Figure 1), GFP-tagged Gyk11 and Gk2 signals clearly overlapped with signals from the MitoTracker dye (Figure 4a), and could be found in mitochondrial fractions (Figure $4 \mathrm{~b}$ ). Treatment with proteinase $\mathrm{K}$ led to diminished Gyk11 and Gk2 signals in the mitochondrial fraction (Figure 4c), indicating that these proteins were localized to the mitochondrial outer membrane. It is interesting to note that ectopic expression of Gyk11 and Gk2 led to dramatic clustering of the mitochondria (Figure 4a, white arrows), which was also observed when Gykl1 was overexpressed in HeLa and NIH3T3 cells (Supplementary Figure S3A and B). In comparison, GFP-Gyk remained in the cytoplasm where no mitochondrial rearrangements could be seen. 
a

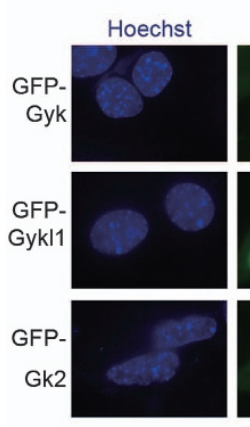

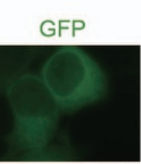
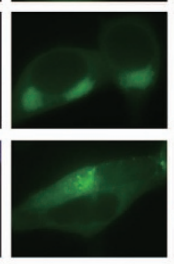
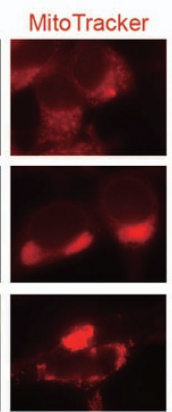

Merge

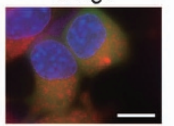

b

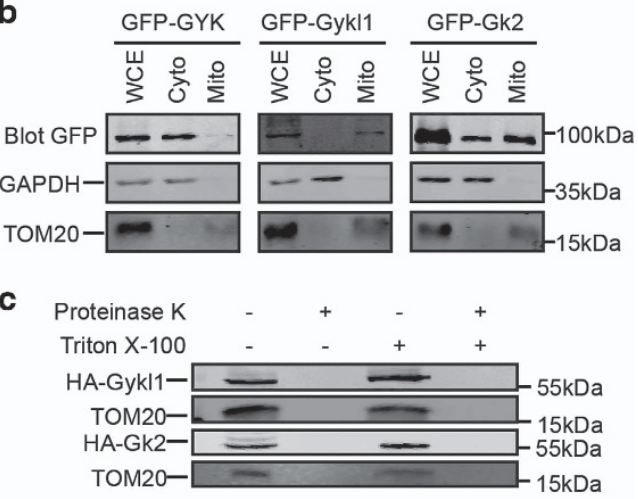

d

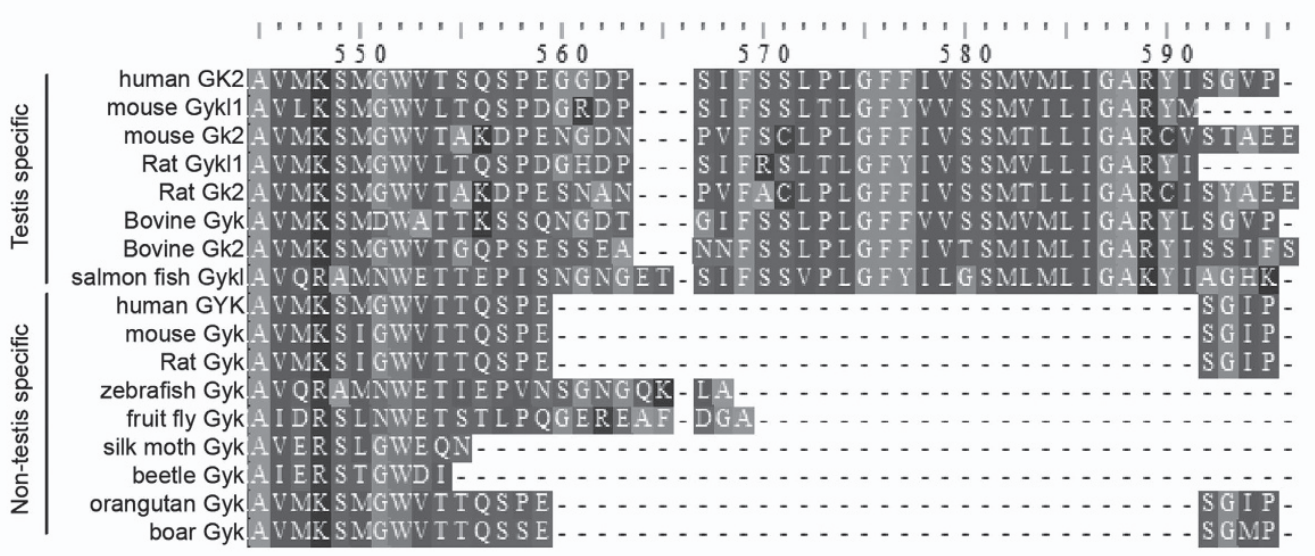

e

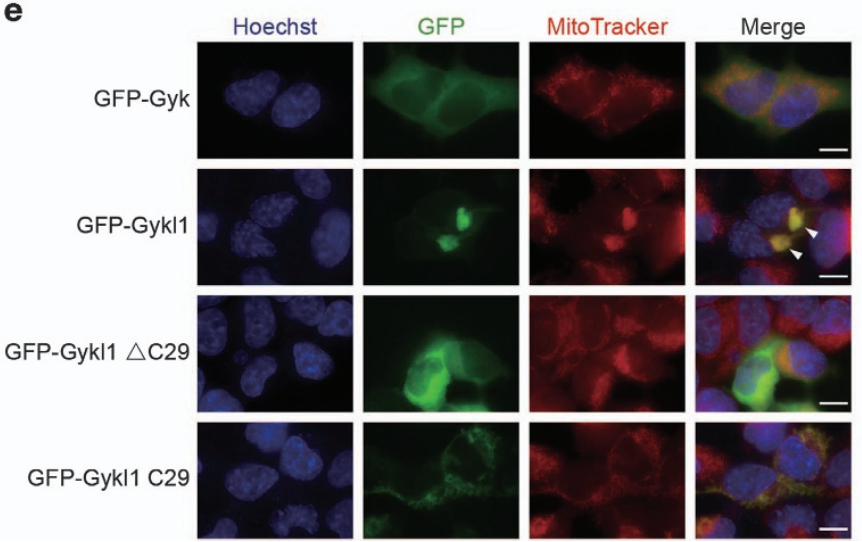

Figure 4 Gykl1 and Gk2 target to the outer membranes of the mitochondria through their unique C-terminal domains. (a) HEK293T cells transiently expressing various GFP-tagged proteins were stained with the MitoTracker dye to visualize the mitochondria. Arrows indicate areas of mitochondrial clustering. Hoechst 33342 was used to visualize the nuclei. Scale bar: $10 \mu \mathrm{m}$. (b) HEK293T cells transiently expressing GFP-tagged proteins were fractionated and blotted with antibodies against GFP. WCE, whole-cell extract. Cyto, cytoplasmic fraction, Mito, mitochondrial fraction. Antibodies against GAPDH and TOM20 served as loading control for the cytosolic and mitochondrial fractions respectively. (c) HEK293T cells transiently expressing HA-tagged Gykl1 and Gk2 were used to isolate mitochondrial fractions. The fractions were treated with proteinase $\mathrm{K}$ in the presence or absence of Triton X-100 before being immunoblotted with anti-HA antibodies. The antibody against TOM20, a mitochondria outer membrane protein, served as a positive control. (d) The C-terminal regions of Gyk-like genes from different species are aligned. Testis-specific genes appear to contain additional sequences in their C-termini compared with Gyk orthologues that are not restricted to testis. (e) HEK293T cells transiently expressing GFP-tagged Gyk as well as full-length and truncation mutants of Gykl1 were stained with the MitoTracker dye to visualize the mitochondria, and examined under a fluorescent microscope. Hoechst 33342 was used to visualize the nuclei. Arrows indicate areas of mitochondrial clustering. Scale bar, $10 \mu \mathrm{m}$. 
Of the 17 Gyk family members and orthologues found in the NCBI, several have been shown to be testis-specific by GEO database (Figure 4d). When aligned against one another, all the testis-specific Gyk members and orthologues (including Gyk11 and Gk2) contain an extra C-terminal tail that is absent in the non-testis-specific Gyk orthologues. To test whether this C-terminal region is important for mitochondrial targeting, we generated GFP-tagged truncation mutants of Gykl1 and determined their localization. As shown in Figure 4e, deletion of the last 29 amino acids of Gykl1 (Gykl1 $\Delta$ C29) abolished its mitochondrial localization, whereas these 29 amino acids alone (Gyk11 C29) were sufficient to target to the mitochondria. Interestingly, no mitochondrial clustering was observed with overexpression of either mutant, indicating that the C-terminus of Gyk11 contains mitochondriatargeting sequences, and the entire Gykll sequence is needed for inducing mitochondrial clustering.

\section{Gykll and Gk2 interact with Pld6 to induce PA \\ production and mitochondrial clustering}

Gyk phosphorylates glycerol to generate glycerol 3phosphate, a precursor of PA, the latter of which has been closely linked to mitochondrial clustering [4, 14]. Gyk, Gyk11, and Gk2 share high homology in protein sequences, but do differ in several amino acids, including the Asp17 catalytic site (Supplementary Figure S1A). Previous work found no glycerol kinase activities in vitro for either Gykl1 or Gk2 [30]. To determine whether glycerol kinase activities were required for Gyk11/Gk2induced mitochondrial clustering, we treated cells with the glycerol kinase inhibitor $\alpha$-thioglycerol before transiently expressing GFP-Gykl1. Even at very high concentrations, $\alpha$-thioglycerol did not affect mitochondrial clustering (Supplementary Figure S4A and B). Moreover, targeting Gyk to the mitochondria, by fusing the C-terminal mitochondrial targeting sequence of Gykl1 to Gyk, failed to induce mitochondrial clustering (Supplementary Figure S4C). These results suggest that Gykll-induced mitochondrial clustering does not require the glycerol kinase activity, and that Gyk11 and Gk2 likely work as adapter proteins in regulating mitochondrial dynamics.

To further probe the mechanisms of Gyk11/Gk2mediated mitochondria regulation, we assessed possible interactions between Gyk11/Gk2 and several proteins known to regulate mitochondrial dynamics. MFN1 and DRP1 can directly control mitochondrial fusion and fission [14]. LIPIN1 was reported to degrade PA and generate DAG during the process of mitochondria fission [16], and $\mathrm{Pa}_{-} \mathrm{pla}_{1}$ can induce mitochondrial fragmentation by turning PA into LPA [1]. Pld6 (MitoPLD) has key roles in generating PA from
CL and mitochondria clustering [4]. GST pull-down experiments found no interactions between Gyk and any of the regulators examined (Supplementary Figure S5A). Neither could we detect interactions of Gyk11/Gk2 with Mfn1, Drp1, Lipin1 or Pa-pla (Supplementary Figure S5B and C). However, both Gyk11 and Gk2, but not Gyk, were able to coprecipitate with Pld6 (MitoPLD) (Figure 5a; Supplementary Figure S5). This interaction appeared to be independent of mitochondrial localization because deleting the mitochondrial localization sequence (MLS) from both Gykl1 and Pld6 (Gyk11 $\Delta$ C29 and Pld6 2 MLS) did not affect their interaction in vitro (Figure 5b). Taken together, these results raise the possibility that the Gyk-like proteins Gyk11/Gk2 may regulate mitochondrial dynamics through Pld6 and the PA biogenesis pathway.

When Pld6 is overexpressed in cells, mitochondrial PA synthesis is upregulated, which leads to increased mitochondrial clustering [4]; Pa-pla 1 , on the other hand, can reduce mitochondrial PA levels, resulting in mitochondrial fragmentation [1]. To determine whether Gyk11/Gk2-induced mitochondrial clustering involves the PA synthesis pathway, we ectopically expressed various proteins in HEK293T cells and monitored intracellular PA levels using GFP-tagged PA-binding domain of Raf1 (GFP-PABD) [40]. As expected, overexpression of Pld6 led to clustered signals of GFP-PABD that also co-stained with MitoTracker (Figure 5c). In contrast, expression of Pa-pla 1 did not alter the distribution of GFP-PABD signals. Similar to Pld6-expressing cells, cells expressing Gyk11 and Gk2, but not Gyk, also displayed elevated GFPPABD signals and clustering in the mitochondria (Figure 5c). If this Gykl1/Gk2-induced mitochondrial clustering involved PA synthesis, then changing mitochondrial PA levels (e.g., through Pld6/Pa-pla expression) should also impact mitochondrial dynamics in Gyk11/Gk2-expressing cells. Indeed, addition of Pld6 or Pa-pla 1 , led to increased or decreased percentages of Gyk11/Gk2-expressing cells showing mitochondrial clustering, respectively (Figure $5 \mathrm{~d}-\mathrm{g}$ ). These results suggest that Gyk11/Gk2 interacts with Pld6, trigger PA synthesis, and induce mitochondrial clustering in cells.

\section{Gyk-like proteins specifically induce mitochondrial clustering via Pld6}

Next we hypothesized if Gyk11/Gk2 could further stimulate PA synthesis and induce mitochondrial clustering when Pld6 is overexpressed. As expected, 
a

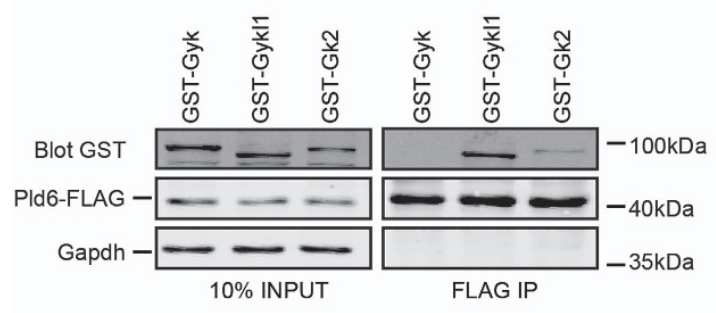

b

MBP-Gykl1 $\triangle \mathrm{MLS}-++\quad+$

+ ++

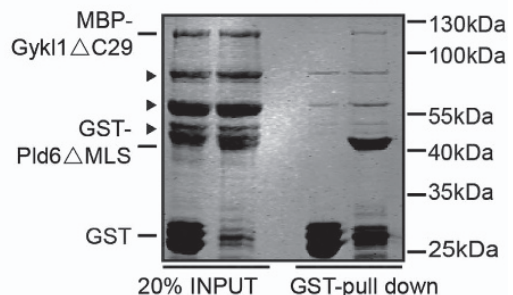

C
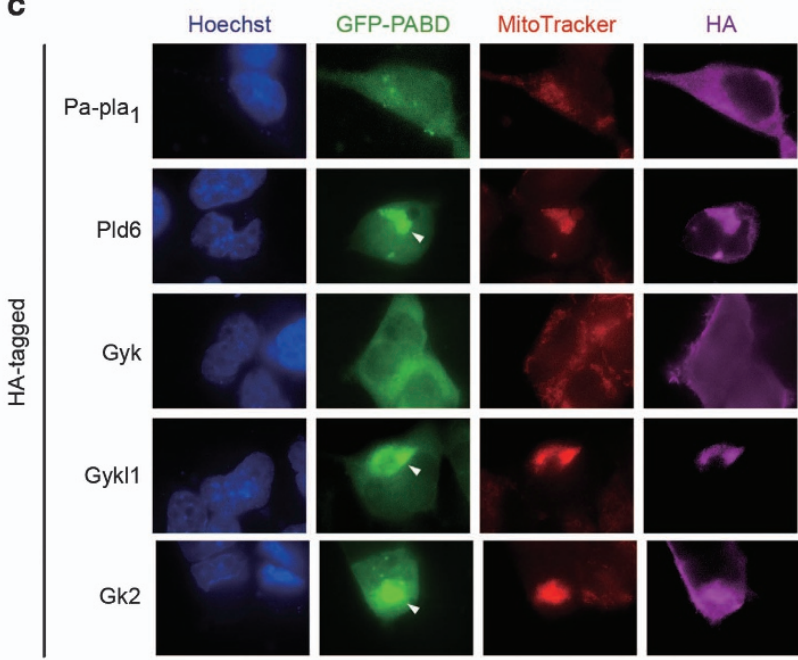

Merge
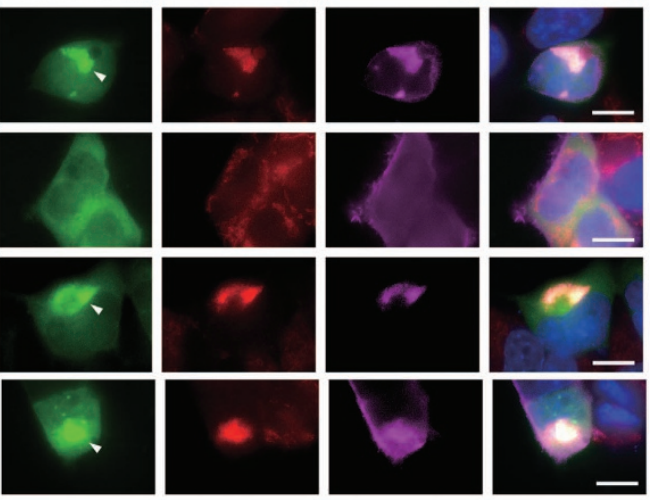

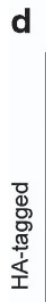
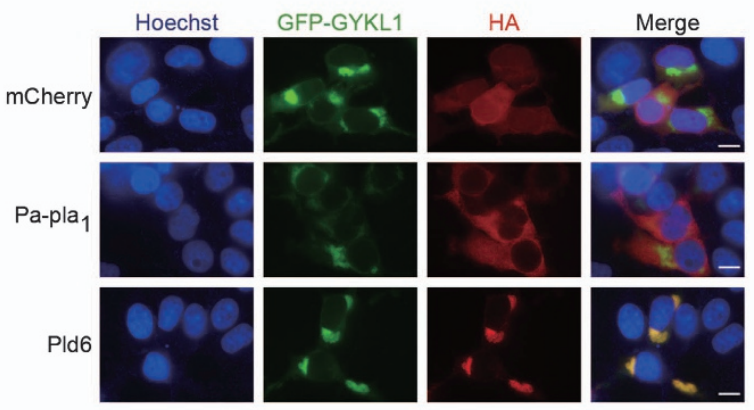

e
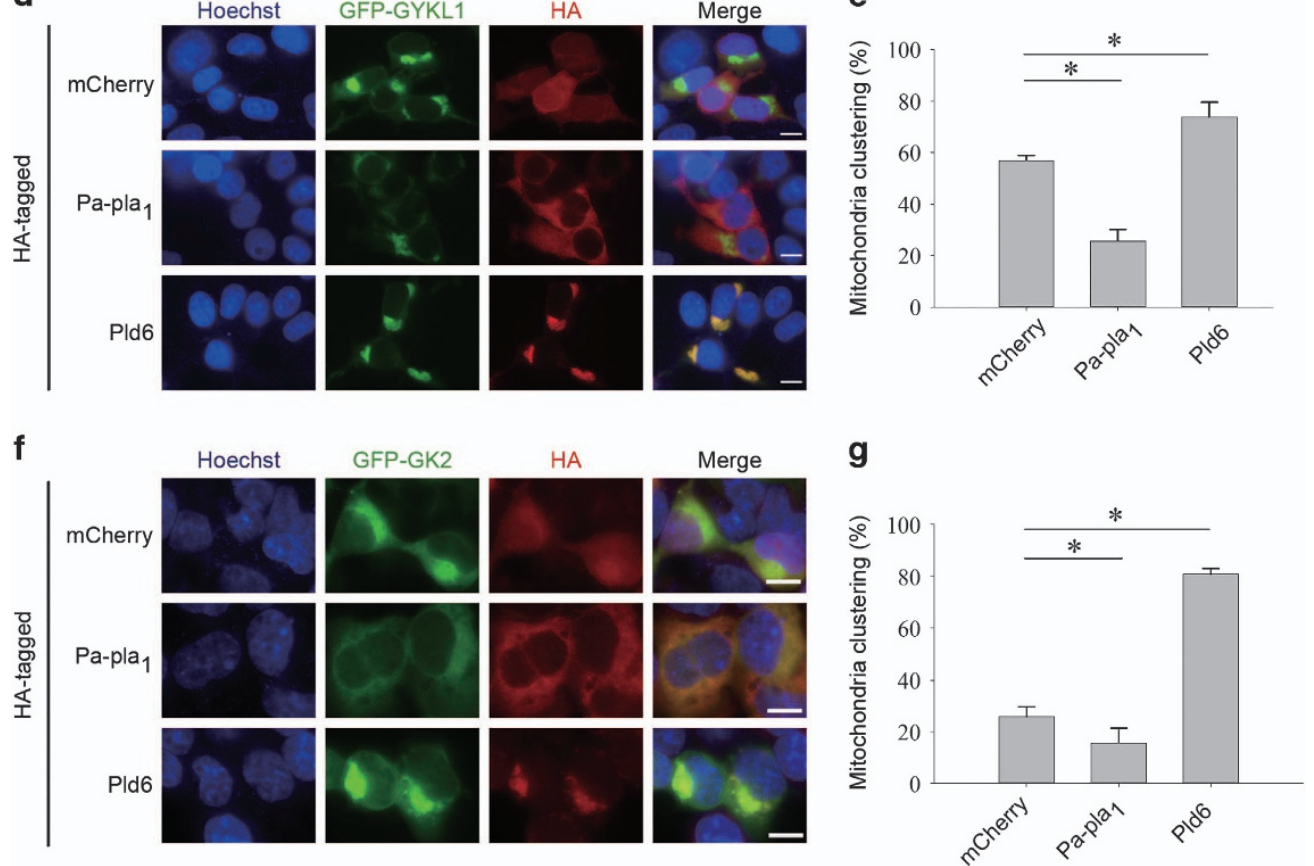
when we reciprocally co-expressed Gyk11 or Gk2 in cells ecto-expressing Pld6, induced mitochondrial clustering was clearly visible $(\sim 70-80 \%)$, compared with cells co-expressing Gyk ( 40\%) (Figure 6a and b). Our results thus far support the notion that Gykl1 and Gk2 can induce mitochondrial clustering through interactions with Pld6 and upregulation of PA synthesis in the mitochondria. We further tested this model by knocking down Pld6 in Gyk11/Gk2-expressing cells. All three siRNAs achieved $>60 \%$ knockdown efficiency (Figure 6c). Importantly, inhibition of Pld6 in these cells diminished GFP-PABD signals in the mitochondria, indicating lower PA levels, and resulted in more diffused staining of MitoTracker, indicating less mitochondrial clustering (Figure $6 \mathrm{~d}-\mathrm{g}$ ). Similar results were also obtained using $\mathrm{HeLa}$ cells overexpressing Gykl1 (Supplementary Figure S6A-C). These results reinforce the notion that Gyk11/Gk2induced mitochondrial clustering and PA synthesis depends on Pld6.

Unlike mouse, human appears to have only one Gyk-like protein-GK2. The human homolog of mouse Gyk11 and Gk2 could also specifically localize to the spermatozoa midpiece (Supplementary Figures S1B and S7A). Furthermore, GK2 could also interact with hPLD6 (Supplementary Figure S7B). As was the case with mouse Gyk11 and Gk2, ectopic expression of GK2 similarly led to changes in mitochondrial dynamics, and this regulation again depended on hPLD6 and involved PA biogenesis in the mitochondria (Supplementary Figure S7C-F), indicating the regulation of PA biosynthesis as central to mitochondrial clustering induced by Gyk-like proteins. Mitochondrial function was also regulated by Gyklike proteins. ATP concentration in Gykl1 or Gk2-defciency spermatozoa was significantly higher than the wild-type one (Supplementary Figure S8A). Cells overexpressing Gyk11, Gk2 or GK2 had decreased ATP level (Supplementary Figure S8B). Regarding that increased GK2 may correlate to asthenozoospermia [32], we suspected that increased GK2 could induce abnormal mitochondrial function or ATP deficiency then contribute to asthenozoospermia. Our studies here provide the first evidence for the evolutionarily conserved functions of Gyk-like proteins, which activate Pld6 to induce mitochondrial PA biogenesis and clustering, processes that are crucial for mitochondrial ATP production, spermiogenesis and male fertility.

\section{Discussion}

Spermatogenesis is essential to sexual reproduction and is highly regulated. Of the numerous genes known to be involved in the process of spermatozoa production, several have testis-specific isoforms with important functions in germ cells [41-44]. Unlike $G y k$, the $G y k$-like intronless retroposons Gykll and Gk2 are expressed primarily in testis [30]. Here, we show for the first time that Gyk11 and Gk2 are in fact localized to the mitochondria in round and elongating spermatids and retained within the mitochondrial sheath in spermatozoa. While Gyk-deficient mice die within 3-4 days after birth, exhibiting postnatal growth retardation, autonomous glucocorticoid synthesis, and abnormal fat metabolism [25], Gykl1 ${ }^{-1-}$ and $G k 2^{-1-}$ mice were viable. However, these mutant mice display specific reproductive defects. For example, Gykl1 ${ }^{+-}$males showed subfertility and $G y k l 1^{-1-}$ males were infertile, and their spermatozoa exhibiting unregulated ATP level and reduced motility. Interestingly, Gykl1 ${ }^{+/-}$male mice were subfertile, while $G k 2^{+/-}$male mice display

Figure 5 Gykl1/Gk2 interacts with Pld6 and stimulate mitochondrial clustering. (a) HEK293T cells transiently co-expressing FLAGtagged Pld6 with GST-tagged Gyk, Gykl1 or Gk2 were immunoprecipitated (IP) with anti-FLAG antibodies. The immunoprecipitates were blotted with antibodies against GST or FLAG. An antibody against GAPDH was used for loading control. (b) Bacterially-purified GSTtagged recombinant PId6 lacking the mitochondria localization sequence (GST-PId6 $\Delta$ MLS), was mixed with purified MBP-tagged recombinant Gykl1 lacking the C-terminal 29 amino acids (MBP-Gykl1 $\Delta$ C29) for in vitro GST pull-down assays. The reaction mixtures were resolved by SDS-PAGE and stained with Coomassie blue. Recombinant GST alone served as a negative control. (c) HEK293T cells transiently co-expressing GFP-PABD with HA-tagged Pa-pla1, Pld6, Gyk, Gykl1 or Gk2 were stained with an antiHA antibody and the MitoTracker dye. Hoechst 33342 was used to visualize the nuclei. PABD is the Raf1 Phosphatidic Acid Binding Domain. Arrows indicate areas of mitochondrial clustering. Scale bar, $10 \mu \mathrm{m}$. (d) HEK293T cells transiently co-expressing GFP-Gykl1 with HA-tagged mCherry, Pld6 or Pa-pla 1 were immunostained with an anti-HA antibody. Hoechst 33342 was used to visualize the nuclei. Scale bar, $10 \mu \mathrm{m}$. (e) Cells from $\mathbf{d}$ were scored for cells with obvious mitochondrial clustering in three independent experiments $(n=3)$ and the percentages of cells with mitochondrial clustering are plotted here. At least 200 cells were counted for each group. Error bars represent s.d. Statistical significance was determined by ANOVA; ${ }^{*} P<0.05$. (f and $\mathbf{g}$ ) HEK293T cells co-expressing GFP-Gk2 with HAtagged mCherry, Pld6, or Pa-pla ${ }_{1}$ were similarly examined (f) and analyzed $(\mathbf{g})$ as described in $\mathbf{d}$ and $\mathbf{e}$. At least three independent experiments were performed with $>200$ cells counted in each group. ${ }^{*} P<0.05$. Scale bar, $10 \mu \mathrm{m}$. 
a
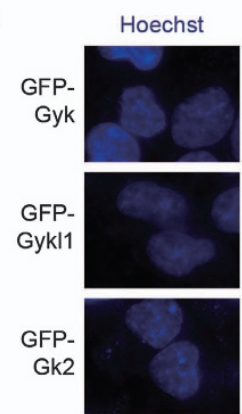

d

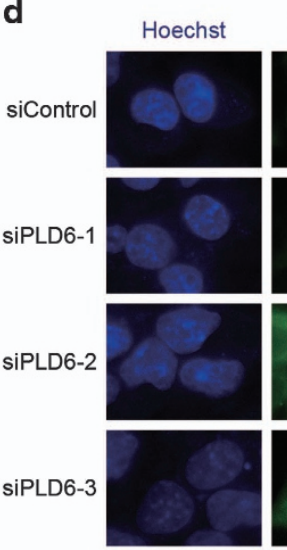

f

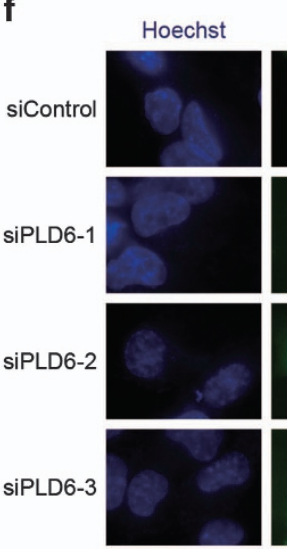

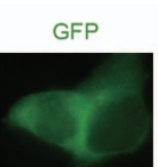
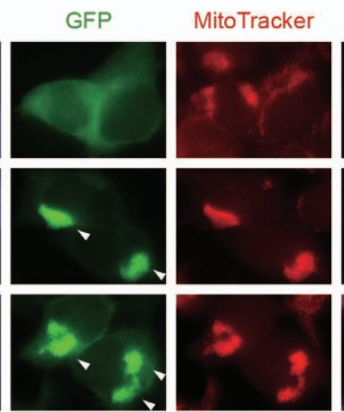

a.
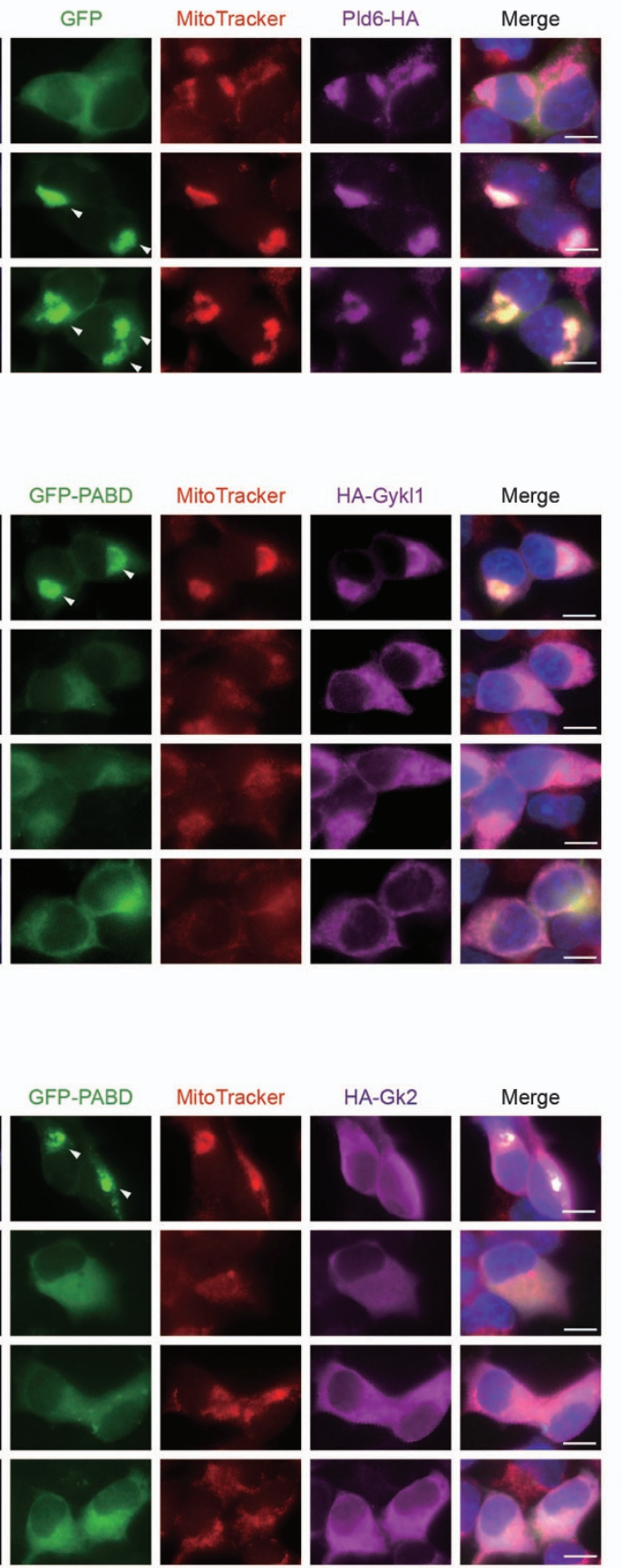

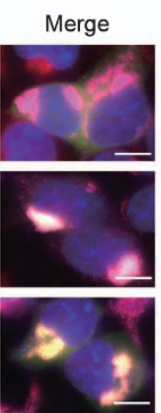

Merge
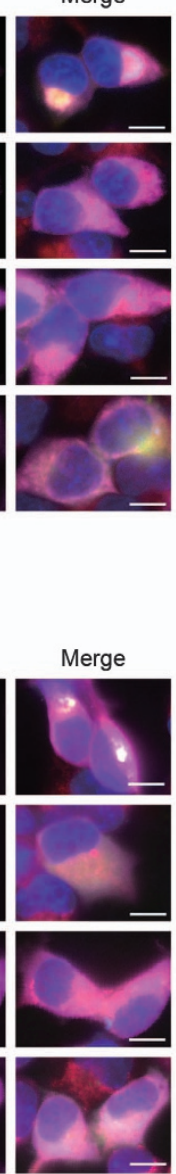

b
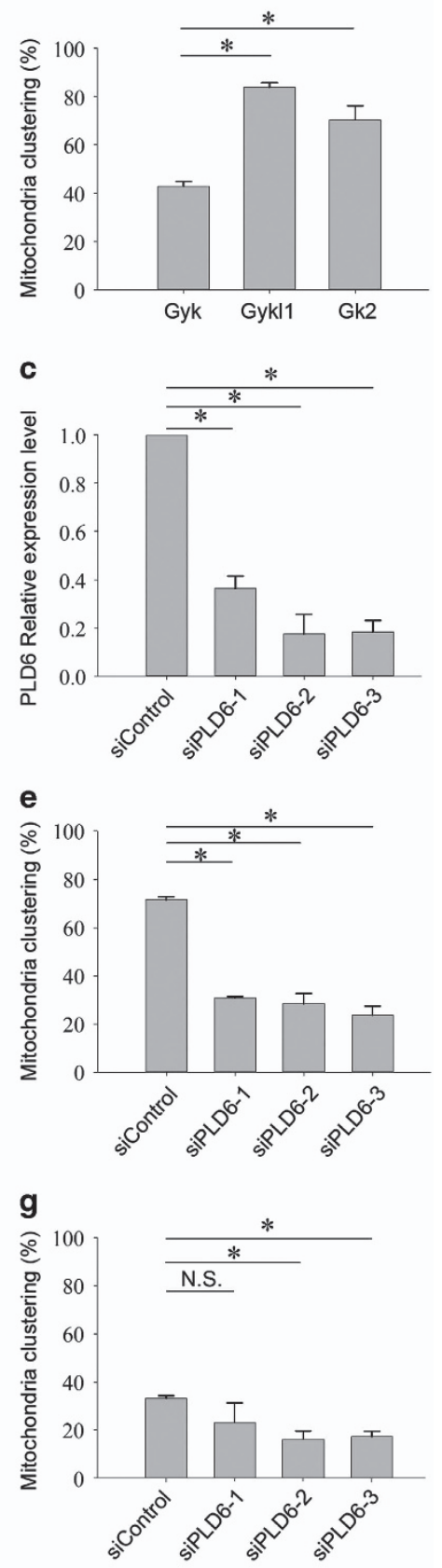

completely normal fertility. Gk2 KO mice showed similar reproductive defects, indicating functional similarities between Gk2 and Gyk11. Still, it needs more in depth investigations to define any function similarity between Gyk11 and Gk2.

Mitochondrial sheath abnormalities in the spermatozoa have been observed in a number of mutant mice, including those deficient for SEPP1, Sept4, KLC3, SLIRP, GPx4, Pa-pla 1 or Guf1 [1, 7, 45-49]. How SEPP1, Sept4, SLIRP and Guf1 maintain mitochondrial sheath integrity remain unclear $[45,46$, $48,49]$. KLC3 has been proposed to regulate mitochondrial trafficking in spermatids [7], and $\mathrm{Pa}_{-} \mathrm{pla}_{1}$ is important in phospholipid metabolism during spermatogenesis [1]. In this study, we have provided evidence that links Gyk11 and Gk2 to mitochondrial clustering and PA generation, and supports the notion that Gyk-like proteins may regulate mitochondrial dynamics through facilitating PA biogenesis (Figure 7) in spermatogenesis. The absence of Gyk11 or Gk2 
greatly altered mitochondrial morphology and dynamics during spermiogenesis, which negatively impacted sperm tail structure and sperm activity (Figure 7). Mechanistically, Gyk11 and Gk2 appear to cooperate with and depend on Pld6 for their function. It will be interesting to determine whether dysfunction of Gyk11 and/or Gk2 disrupts the interactions between Gyk11/Gk2 and Pld6 and contributes to the spermatozoa defects.

Mitochondrial dynamics is intimately linked to PA synthesis, which occurs through the hydrolysis of CL, phosphorylation of DG, or acylation of LPA. LPA is generated from acylation of glycerol 3-phosphate, a product of Gyk-mediated glycerol phosphorylation $[1,34,50-52]$. The exact roles that PA and LPA have in the pathways by which Gyk-like proteins modulate mitochondrial dynamics remain to be uncovered. In this study, we found that inhibiting glycerol kinase activities in cells had no effects on Gykl1-mediated mitochondrial clustering. This result is supported by the findings of an absence of male reproductive defects in mice deficient in GPAT4, the testis-specific glycerol 3-phosphate acyltransferase that converts glycerol 3-phosphate into LPA [53-55]. These data combined suggest that glycerol kinase activities are not required for the regulation of mitochondrial dynamics by Gyklike proteins.

Testis-specific $G y k$-like genes have been identified in multiple species, although humans have only one (GK2) vs two for mice and rats. We have found evolutionary conservation in both sequence and function for human and mouse homologs, such as the interaction with Pld6 and the regulation of mitochondrial dynamics. While mouse Gyk11 and Gk2 appear to carry out similar function, they are both indispensable since knocking out either gene affected male fertility. How these two proteins with high sequence identity, overlapping localization and shared functionality complement each other during spermatogenesis warrants further investigation. Such studies will also have important implications for humans because GK2 likely assumes the roles of both mGYKL1 and mGK2. Whether this is the case or other as yet unidentified factors that functionally complement GK2 do exist should be examined in more detail. Notably, dysregulated GK2 expression has been linked to teratozoospermia and asthenozoospermia [31, 32]. Defects in mitochondrial sheath and damages in sperm tail in Gykll or Gk2 KO mice mimic the case in GK2related teratozoospermia. Also, the unregulated ATP level we observed indicates a potential mechanism of GK2-related asthenozoospermia that could be partially caused by defects in mitochondrial ATP production. Our findings here point to GK2 as an important player in male fertility. It still needs further investigation on whether GK2 has unexpected enzymatic activities. In depth study on the role of GK2 in spermatogenesis may provide new insights into male fertility.

\section{Materials and Methods}

\section{Plasmid construction}

The cDNAs of full-length Mfn1, Drp1, Lipin1, Pa-pla1, Pld6, $G y k, G y k l 1, G k 2$ and $G K 2$ were cloned into the pENTR/DTOPO via the NotI and AscI restriction sites. Various trunca-

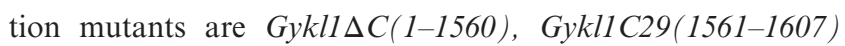
and Pld6 $M L S(115-663)$. The cDNAs were Gateway cloned into various mammalian expression vectors containing Flag-HA tag (pLentiFHA or pMSCVFHA), SFB tag (S-tag, streptavidinbinding protein, Flag) (pLentiSFB or pBabeSFB), GST tag

Figure 6 Gykl1/Gk2-mediated mitochondrial clustering is PA dependent. (a) HEK293T cells transiently co-expressing HA-tagged PId6 with GFP-tagged Gyk, Gykl1 or Gk2 were stained with an anti-HA antibody and the MitoTracker dye. Hoechst 33342 marks the nuclei. Arrows indicate areas of mitochondrial clustering. Scale bar, $10 \mu \mathrm{m}$. (b) Cells from a were scored for mitochondrial clustering and plotted for the percentage of cells with mitochondrial clustering. Three independent experiments were carried out with $>200$ cells per group. Error bars represent s.d. Statistical significance was determined by ANOVA; ${ }^{*} P<0.05$. (c) HEK293T cells were transfected with three different siRNA oligos against Pld6. At $45 \mathrm{~h}$ post transfection, the cells were harvested for RT-qPCR analysis. A scramble siRNA was used as a negative control. The expression level of PId6 was calculated for each group and plotted. Error bars represent s.d. $(n=3)$. Statistical significance was determined by ANOVA; ${ }^{*} P<0.05$. (d) HEK293T cells co-expressing GFP-PABD and HA-Gykl1 were transfected with the PId6 siRNAs from $\mathbf{c}$. At $45 \mathrm{~h}$ post transfection, the cells were harvested for staining using antibodies against $\mathrm{HA}$ and the MitoTracker dye. Hoechst 33342 marks the nuclei. Arrows indicate areas of mitochondrial clustering. Scale bar, $10 \mu \mathrm{m}$. (e) Cells from d were scored and plotted as described in $\mathbf{b}$. Three independent experiments were carried out with $>200$ cells per group. Error bars represent s.d. Statistical significance was determined by ANOVA; ${ }^{*} P<0.05$. (f and $\mathbf{g}$ ) HEK293T cells co-expressing GFP-PABD and HAGk2 were similarly transfected with Pld6 siRNAs, and examined (f) and analyzed $(\mathbf{g})$ as described in $\mathbf{d}$ and $\mathbf{e}$. Three independent experiments were carried out with $>200$ cells per group. Error bars represent s.d. Statistical significance was determined by ANOVA. ${ }^{\star} P<0.05$; Scale bar: $10 \mu \mathrm{m}$. 

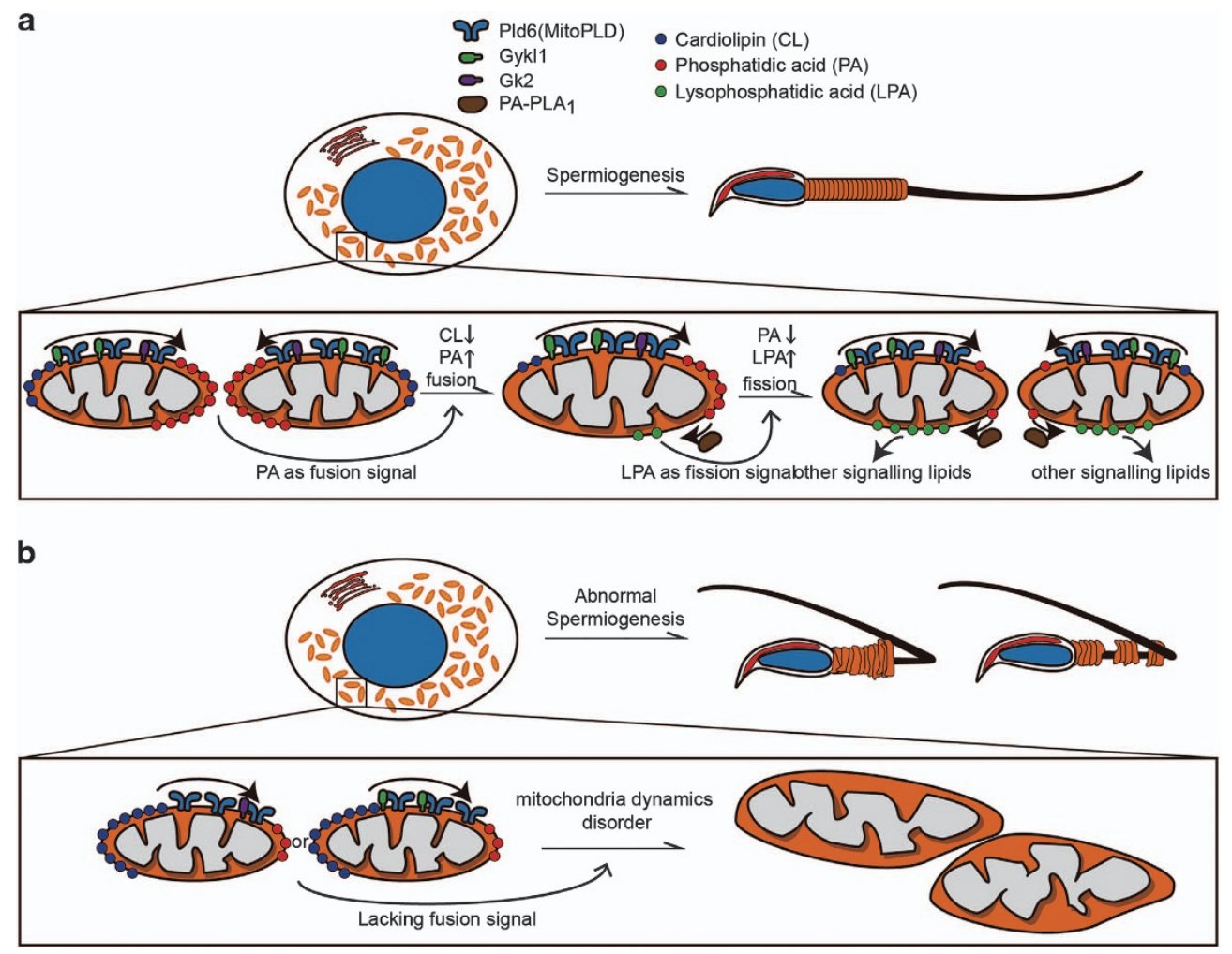

Figure $7 \mathrm{~A}$ model for the regulation of mitochondrial dynamics in spermiogenesis. (a) During normal spermiogenesis, Pld6 (MitoPLD) generates PA from CL. Gykl1 is expressed in round spermatids. Gykl1 and Gk2 physically interact with Pld6 and facilitate Pld6-mediated PA biogenesis, which further induces mitochondrial fusion. Mitochondrial PA is then converted into LysoPA by Pa-pla 1 , which induces mitochondrial fission. PA, LysoPA and other signaling lipids together regulate mitochondrial dynamics and contribute to mitochondrial sheath formation during spermiogenesis. (b) In spermatids lacking Gykl1 or Gk2, PA, LPA and other lipids that regulate mitochondria dynamics are reduced, which contributes to disordered mitochondrial morphology, abnormal regulation of mitochondrial dynamics and disrupted midpiece formation.

(pDEST27), or GFP tag (pHAGE-GFP). Raf1-PABD(11651269) was cloned and ligated to IRES with Pa-plal, Pld6, Gyk, Gykll and GK2.

\section{Generation of genetically-modified mice}

Mice were housed under climate-controlled $\left(22 \pm 1^{\circ} \mathrm{C}\right)$ conditions in a designated pathogen-free animal facility with a 14-hlight/10-h-dark cycle at Sun Yat-Sen University. The Institutional Animal Care and Use Committee of Sun Yat-Sen University, P.R. China have approved all the experimental protocols concerning the handling and husbandry of the mice.

\section{Generation of Gykll and Gk2 knockout mice by CRISPR/ Cas9}

The pT7-3XFlag-hCas9 plasmid was linearized with PmeI for the synthesis of Cas9 mRNA using the mMACHINE T7 ULTRA kit (Life Technologies, Waltham, MA, USA). The pDR274 vector encoding the desired gRNA sequences (Gykll gRNA: AGCGGGAAACTACGATAGTC; $G k 2$ gRNA: TTGGAAGGCGTGCCAATATC) was in vitro transcribed using the MEGAshortscript T7 kit (Life Technologies). The Cas 9 mRNA and the gRNAs were subsequently purified with the MEGAclear kit (Life Technologies), resuspended in RNase- free water, and quantified using NanoDrop-1000. The Cas9 mRNA and gRNA mixture was then injected into 0.5-day zygotes of C57BL/6J mice. Three hours after injection, the injected zygotes were transplanted into the oviduct of 0.5 -day pseudopregnant mothers. CRISPR/Cas9 knockout mice were bred with wild-type mice for more than three generations to minimize off-target effects. Mouse genotyping was done by PCR and sequencing of tail-snips using the Mouse Genotyping Kit (KAPA biosystems, KK 7302) following the manufacturer's instruction.

\section{Immunoblotting}

For tissues samples, tissues were homogenized in ice-cold RIPA buffer (50 mu Tris- $\mathrm{HCl} \mathrm{pH} 8.0,150 \mathrm{~mm} \mathrm{NaCl}, 1 \% \mathrm{NP}-$ -40, 1 mm EDTA pH 8.0, 0.5\% sodium deoxycholate, $0.1 \%$ SDS) supplemented with protease inhibitor cocktail (Sigma, St Louis, MO, USA, P8340). The homogenates were clarified by centrifugation and quantified with BCA Protein Assay Kit (Pierce, Waltham, MA, USA, 23225). For cultured cells and protein, $1 \times$ SDS-PAGE loading buffer was added and heated at $95^{\circ} \mathrm{C}$ for $20 \mathrm{~min}$. Sample were then subjected to SDS-PAGE and transferred to membrane. After blocking by $5 \%$ non-fat milk, proteins were detected with antibodies. Rabbit anti-Gyk11/ 
Gk2 antibody (1:10 000), rabbit anti-GST antibody (1:10 000), mouse anti-GAPDH antibody (1:5000), mouse anti-TOM20 antibody (1:5000), rabbit anti-HA antibody (1:5000), mouse anti-FLAG antibody (1:5000), rabbit anti-DYKDDDDK antibody (1:5000), goat anti-mouse IgG (1:10 000), goat antirabbit $\operatorname{IgG}(1: 10000)$. The membrane was scanned using the Licor Odyssey system.

\section{Histological analysis}

For hematoxylin and eosin staining, Bouin's solution-fixed and paraffin-embedded testis sections $(4 \mu \mathrm{m})$ were sequential rehydrated and stained with hematoxylin and eosin.

\section{Immunofluorescent microscopy}

For testis sections, testis sections were rehydrated and microwaved for $10 \mathrm{~min}$ in citric buffer $(10 \mathrm{~mm}$ citric acid monohydrate, $10 \mathrm{~mm}$ sodium citrate). For spermatozoa, spermatozoa was diluted in $\mathrm{H}_{2} \mathrm{O}$ containing $150 \mathrm{nM}$ Mitotracker Red CMXRos at $25^{\circ} \mathrm{C}$ for $15 \mathrm{~min}$. The spermatozoa was speared and dried on a slide, then fixed with $4 \%$ formaldehyde in PBS at $25^{\circ} \mathrm{C}$ for $30 \mathrm{~min}$. For adhered cells, samples were fixed in $4 \%$ formaldehyde in PBS at $25^{\circ} \mathrm{C}$ for $30 \mathrm{~min}$. Permeabilize by $0.5 \%$ Trition-X, $20 \mathrm{~mm}$ HEPES, $50 \mathrm{~mm} \mathrm{NaCl}, 3 \mathrm{~mm} \mathrm{MgCl}_{2}$, $300 \mathrm{~mm}$. Then samples were incubated with primary antibodies in PBS ( $\mathrm{pH} 7.4$ ) containing 5\% BSA for $1 \mathrm{~h}$ at room temperature. After three times washes by PBS, the samples were incubated with secondary antibodies in PBS ( $\mathrm{pH} 7.4$ ) containing 5\% BSA and $10 \mu \mathrm{g} \mathrm{ml}^{-1}$ Hoechst 33342 (Invitrogen, H3570) for $1 \mathrm{~h}$ at room temperature. Antibodies and dilutions: rabbit antiGyk11/Gk2 antibody (1:1 000), mouse anti-SYCP3 (1:200), goat anti-PLZF antibody (1:500), mouse anti-HA antibody (1:1 000), Alexa Fluor 488-conjugated donkey anti-mouse IgG (1:2 000), Alexa Fluor 488-conjugated donkey anti-rabbit IgG (1:2 000), Alexa Fluor 555-conjugated donkey anti-mouse IgG (1:2 000), Alexa Fluor 555-conjugated donkey anti-rabbit IgG (1:2 000), Alex Fluor 647-conjugated donkey anti-mouse IgG (1:1000) and Alexa Fluor 647-conjugated donkey anti-goat IgG (1:1 000, Life Technologies).

\section{Breeding analysis}

Indicated numbers of 5 month old WT and KO male mice were subjected for breeding analysis. Each male mouse mated with a WT female (8 weeks) mouse until observation of vaginal plug. Litter number was counted 14-24 days after vaginal plug formation. Any offspring producing was marked as successful pregnancy.

\section{Epididymal sperm analysis}

The cauda epididymis was obtained from 19-week-old mice. Sperm from the cauda epididymis was collected into $3 \%$ BSA in DMEM/F-12 (Gibco, Waltham, MA, USA, 11039-021) at $37^{\circ} \mathrm{C}$. Sperm motility was monitored using a computer-assisted sperm analysis system (Sperm Class Analyzer, MICROPTIC S.L.).

\section{Electron tomography and image processing}

Electron tomography of the semi-ultrathin sections was conducted using Tomography in the FEI Tecnai F20 microscope (FEI, Hillsboro, OR, USA) operated at $200 \mathrm{kV}$. The data were recorded with a $4 \mathrm{~K} \times 4 \mathrm{~K} \mathrm{CCD}$ camera (Eagle, FEI, USA), and the tilt angles ranged from $-60^{\circ}$ to $+60^{\circ}$ with a $2^{\circ}$ interval. The nominal magnifications of the tomograms were $7800 \times, 11500 \times$ and $14500 \times$, and the corresponding final $\AA$ / pixel values were 15.4, 19.6 and 28.3, respectively. The IMOD package (Kremer et al., 1996) was used for reconstruction, segmentation and rendering.

\section{Intracytoplasmic spermatozoa injection}

Six-week-old female mice were superovulated with PMSG and hCG. Unfertilized oocytes were harvested $14 \mathrm{~h}$ after injection of hCG. ${ }^{-1-}$ Sperm heads were then injected into the cytoplasm of the unfertilized oocytes by microinjection. The fertilized oocytes were cultured overnight in KSOM medium. The next day, 2-cell embryos were transplanted into a pseudopregnant mother.

\section{Mitochondrial fractionation}

Mitochondria were prepared as previously described (Wieckowski, et al., 2009). Briefly, cells were harvested and washed with PBS before addition of ice-cold hypotonic buffer (225 mm mannitol, $75 \mathrm{~mm}$ sucrose, $0.05 \mathrm{~mm}$ EDTA, $30 \mathrm{~mm}$ Tris$\mathrm{HCl}, \mathrm{pH}$ 7.4). The cells were then homogenized by 75 strokes of a Telflon homogenizer. Unbroken cells and nuclei were removed by centrifugation at $600 \mathrm{~g}$ for $5 \mathrm{~min}$ at $4{ }^{\circ} \mathrm{C}$. Mitochondrial and cytosolic fractions were separated by centrifugation at $9000 \mathrm{~g}$ for $10 \mathrm{~min}$ at $4{ }^{\circ} \mathrm{C}$. The resulting mitochondria were treated in the presence or absence of $0.5 \%$ Triton $\mathrm{X}-100$ or $100 \mu \mathrm{g}$ protease $\mathrm{K}$ on ice for $30 \mathrm{~min}$. The reaction was stopped by adding $5 \times$ SDS-PAGE loading buffer for further analysis.

\section{$R T-P C R$ and quantitative $R T-P C R$}

Total RNA from various mouse tissues were extracted with TRIzol reagent (Invitrogen, Waltham, MA, USA, 15596-018). First-strand cDNAs were synthesized using RevertAid First Strand cDNA Synthesis Kit (Thermo Scientific, Waltham, MA, USA, \#K1621). RT-PCR was performed using TAKARA Taq (TAKARA, Kusatsu, Shiga, Japan, R001A). Reaction mixture was heated at $95^{\circ} \mathrm{C}$ for $3 \mathrm{~min}$, followed by 25 cycles of denaturing at $95^{\circ} \mathrm{C}$ for $20 \mathrm{~s}$, annealing at $60^{\circ} \mathrm{C}$ for $30 \mathrm{~s}$ and extension at $72{ }^{\circ} \mathrm{C}$ for $45 \mathrm{~s}$ with a final extension at $72{ }^{\circ} \mathrm{C}$ for $5 \mathrm{~min}$.

RT-PCR primers: Gyk RT FP: CTACAAGGGCAGG TTGAGTT, Gyk RT RP: GATGGTCTGTGCATGGTGGC, Gykl1 RT FP: TGCGGGCTCACCCAGTT, Gyk11 RT RP: CGACCTTCACTTTCCTCGACAT, Gk2 RT FP: AAATGT TTGGAGTCTTGAACCTGAA, Gk2 RT RP: GTATTTGTA GGGGCCTCATGGGTTC, mouse $\beta$-actin RT FP: GTCCC TCACCCTCCCAAAAG, mouse $\beta$-actin RT RP: GCTGCC TCAACACCTCAACCC.

Quantitative PCR was performed using GoTaq qPCR Master Mix (Promega, Madison, WI, USA, A6002). Reaction mixture was heated at $95^{\circ} \mathrm{C}$ for $10 \mathrm{~min}$. 40 cycles amplification of $95^{\circ} \mathrm{C}$ for $15 \mathrm{~s}$ and $60^{\circ} \mathrm{C}$ for $60 \mathrm{~s}$. Data were analyzed by The StepOnePlus ${ }^{\mathrm{TM}}$ System (ABI).

Quantitative PCR primers: Gykl1 q FP: TGCGGGC TCACCCAGTT; Gykl1 q RP: CGACCTTCACTTTCCT CGACAT mGapdh q FP: CATGAGAAGTATGACAACA 
GCCT, mGapdh q RP: AGTCCTTCCACGATACCAAAGT, hPLD6 q FP: TCGTCACCGACTGCGACTA, hPLD6 q RP: CACGATGGCAAACTTGTGATG hGAPDH-q-FP: CATG AGAAGTATGACAACAGCCT, hGAPDH-q-RP: AGTCCT TCCACGATACCAAAGT.

\section{Cell culture, transfection and coimmunoprecipitation}

HEK293T, HeLa and NIH3T3 cells were cultured in DMEM with $4.5 \mathrm{~g}^{-1}$ glucose, L-glutamine and sodium pyruvate (CORNING, 10-013-CVR) with 10\% fetal bovine serum under standard condition. Plasmids were transfected using PEI (P3143, Sigma) or Lipofectamine 2000 (Invitrogen, 11668-019) according to manufacturer's instruction. Cells were collected and lysed in ice-cold IP buffer (50 mM Tris- $\mathrm{HCl}, \mathrm{pH} 8.0,100 \mathrm{~mm}$ $\mathrm{NaCl}, 1$ mм EDTA, 0.5\% NP-40, 10\% Glycerol) supplemented with protease inhibitor cocktail at $4{ }^{\circ} \mathrm{C}$ for $30 \mathrm{~min}$. Cell lysis was clarified and incubated with M2-affinity gel (A2220-5ML) at $4^{\circ}$ $\mathrm{C}$ for $60 \mathrm{~min}$. The gel was washed and eluted by $1 \times$ SDS-PAGE loading buffer for immunoblotting analysis.

\section{$R N A$ interference}

The siRNA targeting sequences used in HeLa or HEK293T cells: siControl: scramble, siPLD6-1: GCTA CATGCATCACAAGTT, siPLD6-2: CGCAAGCCATCC AGAACAA, siPLD6-3: CAGCGAAAGCCAAACCTAA (RIBOBIO). Final concentration of $50 \mathrm{nM}$ of siRNA was used to transfect HeLa or HEK293T cells in a reverse transfection manner according to manufacturer's instruction. Cells were collected or fixed $45 \mathrm{~h}$ post-transfection for further analysis.

\section{Protein purification}

mPLD6 $\Delta$ MLS(115-663) was sub-cloned into a modified pGEX5-2 vector through NotI and AscI restriction sites. The resulting plasmid was then transformed into BL21 (DE3). The GST fusion protein was expressed by adding $0.1 \mathrm{~mm}$ IPTG, and the bacteria were further cultured at $16^{\circ} \mathrm{C}$ for $16 \mathrm{~h}$ until the OD600 reached 1.0. GST-mPLD6 2 MLS was purified with a Glutathione Sepharose 4B column (GE Healthcare, 17-0756-01) in lysis buffer (50 mm Tris- $\mathrm{HCl}, \mathrm{pH} \quad 8.0,300 \mathrm{~mm} \mathrm{NaCl})$ according to the manufacturer's instructions. Gyk11 $\Delta \mathrm{C} 29$ (1-1 560) was sub-cloned into a modified pDest17 vector containing an MBP tag through NotI and AscI restriction sites. The resulting plasmid was then transformed into BL21 (DE3). The His-MBP fusion protein was expressed by adding $0.1 \mathrm{~mm}$ IPTG, and the bacteria were further cultured at $16^{\circ} \mathrm{C}$ for $16 \mathrm{~h}$ until the OD600 reached 1.0. His-MBP-Gyk11 $\Delta \mathrm{C} 29$ was purified through a Ni Sepharose FF column (GE Healthcare, Pittsburgh, PA, USA, 17-5318-01) in lysis buffer 2 (50 mm Tris- $\mathrm{HCl}, \mathrm{pH} 8.0$, $300 \mathrm{~mm} \mathrm{NaCl}, 20 \mathrm{~mm}$ imidazole) according to the manufacturer's instructions. The purified proteins were mixed at $4{ }^{\circ} \mathrm{C}$ for $12 \mathrm{~h}$. The mixture was then clarified by centrifugation and incubated with Glutathione Sepharose $4 \mathrm{~B}$ for $30 \mathrm{~min}$. The resin was washed with lysis buffer (50 mm Tris- $\mathrm{HCl}, \mathrm{pH} 8.0,300 \mathrm{~mm}$ $\mathrm{NaCl}$ ). Protein was eluted by $1 \times$ SDS-PAGE loading buffer for SDS-PAGE and Coomassie staining.

\section{ATP level determination}

The ATP values of cells were determined using Beyotime Enhanced ATP Assay Kit (S0027). The results were normalized to cell numbers of the samples.

\section{Statistical analysis}

The statistical significances between the mean values for different samples were examined using one-way ANOVA with the Holm-Sidak test when the data were normally distributed with equal variance (SigmaPlot 12.5). Otherwise, KruskalWallis ANOVA on ranks was used (SigmaPlot 12.5). Percentage data were arc-sine-transformed before testing. The data were considered significant when $P<0.05 \quad(*), 0.01 \quad(* *)$ or $0.001(* * *)$.

\section{Conflict of Interest}

The authors declare no conflict of interest.

\section{Acknowledgements}

We thank Dr. Dangsheng Li for comments on the manuscript. This work was supported by National Key R\&D Program of China (2017YFC1001901 and 2017YFA0102800), National Natural Science Foundation of China (grant numbers 31671540, 31371508, 31401223, 81330055 and 31601196); Natural Science Foundation of Guangdong Province (2016A030310206); Guangdong Science and Technology Department Planning Project (grant numbers 2015A020212010, 2015A020212005 and 2015B020228002); Zhujiang Program of Science and Technology Nova in Guangzhou (201710010042); Fundamental Research Funds for the Central Universities (Sun Yat-sen University) (grant numbers 161gpy30, 161gpy31 and 161gzd13; NCI CA211653, CPRIT RP160462, and the Welch Foundation Q-1673).

\section{Author contributions}

$\mathrm{ZS}$ and $\mathrm{JH}$ conceived the study. YC, PL, YH and $\mathrm{JH}$ designed the overall experimental scheme. YC, PL, ML, XZ, $\mathrm{CD}, \mathrm{JF}, \mathrm{ZZ}, \mathrm{XZ}, \mathrm{SC}, \mathrm{HZ}$ and $\mathrm{YG}$ executed the experiments. YC, QZ, ZS and JH analyzed the data. YC, YH, DL and JH wrote the paper.

\section{References}

1 Baba T, Kashiwagi Y, Arimitsu N et al. Phosphatidic acid (PA)-preferring phospholipase A1 regulates mitochondrial dynamics. J Biol Chem 2014; 289: 11497-11511.

2 Youle RJ, van der Bliek AM. Mitochondrial fission, fusion, and stress. Science 2012; 337: 1062-1065.

3 Mishra P, Chan DC. Mitochondrial dynamics and inheritance during cell division, development and disease. Nat Rev Mol Cell Biol 2014; 15: 634-646.

4 Choi SY, Huang P, Jenkins GM, Chan DC, Schiller J, Frohman MA. A common lipid links Mfn-mediated 
mitochondrial fusion and SNARE-regulated exocytosis. Nat Cell Biol 2006; 8: 1255-1262.

5 Manifava M, Thuring JW, Lim ZY, Packman L, Holmes AB, Ktistakis NT. Differential binding of traffic-related proteins to phosphatidic acid- or phosphatidylinositol $(4,5)$ bisphosphate-coupled affinity reagents. J Biol Chem 2001; 276: 8987-8994.

6 Tanaka Y, Kanai Y, Okada Y et al. Targeted disruption of mouse conventional kinesin heavy chain, kif5B, results in abnormal perinuclear clustering of mitochondria. Cell 1998; 93: 1147-1158.

7 Zhang Y, Ou Y, Cheng M, Saadi HS, Thundathil JC, van der Hoorn FA. KLC3 is involved in sperm tail midpiece formation and sperm function. Dev Biol 2012; 366: 101-110.

8 Watanabe T, Chuma S, Yamamoto Y et al. MITOPLD is a mitochondrial protein essential for nuage formation and piRNA biogenesis in the mouse germline. Dev Cell 2011; 20: 364-375.

9 Song Z, Ghochani M, McCaffery JM, Frey TG, Chan DC. Mitofusins and OPA1 mediate sequential steps in mitochondrial membrane fusion. Mol Biol Cell 2009; 20: 3525-3532.

10 Meeusen S, DeVay R, Block J et al. Mitochondrial innermembrane fusion and crista maintenance requires the dynamin-related GTPase Mgm1. Cell 2006; 127: 383-395.

11 Otera H, Wang C, Cleland MM et al. Mff is an essential factor for mitochondrial recruitment of Drp1 during mitochondrial fission in mammalian cells. J Cell Biol 2010; 191: 1141-1158.

12 Loson OC, Song Z, Chen H, Chan DC. Fis1, Mff, MiD49, and MiD51 mediate Drp1 recruitment in mitochondrial fission. Mol Biol Cell 2013; 24: 659-667.

13 Ishihara N, Nomura M, Jofuku A et al. Mitochondrial fission factor Drp1 is essential for embryonic development and synapse formation in mice. Nat Cell Biol 2009; 11: 958-966.

14 Huang H, Frohman MA. Lipid signaling on the mitochondrial surface. Biochim Biophys Acta 2009; 1791: 839-844.

15 Gao Q, Frohman MA. Roles for the lipid-signaling enzyme MitoPLD in mitochondrial dynamics, piRNA biogenesis, and spermatogenesis. BMB Rep 2012; 45: 7-13.

16 Huang $\mathrm{H}$, Gao Q, Peng X et al. piRNA-associated germline nuage formation and spermatogenesis require MitoPLD profusogenic mitochondrial-surface lipid signaling. Dev Cell 2011; 20: 376-387.

17 Ramalho-Santos J, Varum S, Amaral S, Mota PC, Sousa AP, Amaral A. Mitochondrial functionality in reproduction: from gonads and gametes to embryos and embryonic stem cells. Hum Reprod Update 2009; 15: 553-572.

18 Otani H, Tanaka O, Kasai K, Yoshioka T. Development of mitochondrial helical sheath in the middle piece of the mouse spermatid tail: regular dispositions and synchronized changes. Anat Rec 1988; 222: 26-33.

19 Ho HC, Wey S. Three dimensional rendering of the mitochondrial sheath morphogenesis during mouse spermiogenesis. Microsc Res Tech 2007; 70: 719-723.
20 Mi Y, Shi Z, Li J. Spata19 is critical for sperm mitochondrial function and male fertility. Mol Reprod Dev 2015; 82: 907-913.

21 Olson GE, Winfrey VP. Mitochondria-cytoskeleton interactions in the sperm midpiece. J Struct Biol 1990; 103: $13-22$.

22 Deng H, Dodson MW, Huang H, Guo M. The Parkinson's disease genes pink1 and parkin promote mitochondrial fission and/or inhibit fusion in Drosophila. Proc Natl Acad Sci USA 2008; 105: 14503-14508.

23 Baklouti-Gargouri S, Ghorbel M, Ben Mahmoud A et al. Identification of a novel $\mathrm{m} .9588 \mathrm{G}>\mathrm{a}$ missense mutation in the mitochondrial COIII gene in asthenozoospermic Tunisian infertile men. J Assist Reprod Genet 2014; 31: 595-600.

24 Kamoshida S, Satoh Y, Kamiya S, Tsutsumi Y. Heat shock protein 60 (HSP60) immunoreactivity in gastric epithelium associated with Helicobacter pylori infection: a pitfall in immunohistochemically interpreting HSP60-mediated autoimmune responses. Pathol Int 1999; 49: 88-90.

25 Huq AH, Lovell RS, Ou CN, Beaudet AL, Craigen WJ. Xlinked glycerol kinase deficiency in the mouse leads to growth retardation, altered fat metabolism, autonomous glucocorticoid secretion and neonatal death. Hum Mol Genet 1997; 6: 1803-1809.

26 Sjarif DR, Ploos van Amstel JK, Duran M, Beemer FA, Poll-The BT. Isolated and contiguous glycerol kinase gene disorders: a review. J Inherit Metab Dis 2000; 23: 529-547.

27 Ohira RH, Dipple KM, Zhang YH, McCabe ER. Human and murine glycerol kinase: influence of exon 18 alternative splicing on function. Biochem Biophys Res Commun 2005; 331: 239-246.

28 Yeh JI, Kettering R, Saxl R et al. Structural characterizations of glycerol kinase: unraveling phosphorylationinduced long-range activation. Biochemistry 2009; 48: 346-356.

29 Sargent CA, Young C, Marsh S, Ferguson-Smith MA, Affara NA. The glycerol kinase gene family: structure of the Xp gene, and related intronless retroposons. Hum Mol Genet 1994; 3: 1317-1324.

30 Pan Y, Decker WK, Huq AH, Craigen WJ. Retrotransposition of glycerol kinase-related genes from the $\mathrm{X}$ chromosome to autosomes: functional and evolutionary aspects. Genomics 1999; 59: 282-290.

31 Platts AE, Dix DJ, Chemes HE et al. Success and failure in human spermatogenesis as revealed by teratozoospermic RNAs. Hum Mol Genet 2007; 16: 763-773.

32 Siva AB, Kameshwari DB, Singh V et al. Proteomics-based study on asthenozoospermia: differential expression of proteasome alpha complex. Mol Hum Reprod 2010; 16: 452-462.

33 Huang Y, Xiong Y, Lin Z et al. Specific tandem 3'UTR patterns and gene expression profiles in mouse Thy1+ germline stem cells. PLOS ONE 2015; 10: e0145417.

34 Francke U, Harper JF, Darras BT et al. Congenital adrenal hypoplasia, myopathy, and glycerol kinase deficiency: molecular genetic evidence for deletions. Am J Hum Genet 1987; 40: 212-227. 
35 Cuasnicu PS, Ellerman DA, Cohen DJ, Busso D, Morgenfeld MM, Da Ros VG. Molecular mechanisms involved in mammalian gamete fusion. Arch Med Res 2001; 32: 614-618.

36 Sugimoto R, Nabeshima Y, Yoshida S. Retinoic acid metabolism links the periodical differentiation of germ cells with the cycle of Sertoli cells in mouse seminiferous epithelium. Mech Dev 2012; 128: 610-624.

37 Li M, Huang R, Jiang X et al. CRISPR/Cas9 promotes functional study of testis specific X-linked gene in vivo. PLoS ONE 2015; 10: e0143148.

38 Liang $\mathrm{P}, \mathrm{Xu} \mathrm{Y}$, Zhang X et al. CRISPR/Cas9-mediated gene editing in human tripronuclear zygotes. Protein Cell 2015; 6: 363-372.

39 Wang H, Yang H, Shivalila CS et al. One-step generation of mice carrying mutations in multiple genes by CRISPR/ Cas-mediated genome engineering. Cell 2013; 153: 910-918.

40 Ghosh S, Strum JC, Sciorra VA, Daniel L, Bell RM. Raf-1 kinase possesses distinct binding domains for phosphatidylserine and phosphatidic acid. Phosphatidic acid regulates the translocation of Raf-1 in 12-Otetradecanoylphorbol-13-acetate-stimulated Madin-Darby canine kidney cells. J Biol Chem 1996; 271: 8472-8480.

41 Escoffier J, Jemel I, Tanemoto A et al. Group X phospholipase A2 is released during sperm acrosome reaction and controls fertility outcome in mice. J Clin Investig 2010; 120: 1415-1428.

42 Sato H, Taketomi Y, Isogai Y et al. Group III secreted phospholipase A2 regulates epididymal sperm maturation and fertility in mice. J Clin Investig 2010; 120: 1400-1414.

43 Cohen DJ, Ellerman DA, Cuasnicu PS. Mammalian spermegg fusion: evidence that epididymal protein DE plays a role in mouse gamete fusion. Biol Reprod 2000; 63: 462-468.

44 Cohen DJ, Rochwerger L, Ellerman DA, Morgenfeld MM, Busso D, Cuasnicu PS. Relationship between the association of rat epididymal protein "DE" with spermatozoa and the behavior and function of the protein. Mol Reprod Dev 2000; 56: 180-188.

45 Kissel H, Georgescu MM, Larisch S, Manova K, Hunnicutt GR, Steller H. The Sept4 septin locus is required for sperm terminal differentiation in mice. Dev Cell 2005; 8: 353-364.

46 Olson GE, Winfrey VP, Nagdas SK, Hill KE, Burk RF. Selenoprotein $\mathrm{P}$ is required for mouse sperm development. Biol Reprod 2005; 73: 201-211.
47 Imai H, Hakkaku N, Iwamoto R et al. Depletion of selenoprotein GPx4 in spermatocytes causes male infertility in mice. J Biol Chem 2009; 284: 32522-32532.

48 Colley SM, Wintle L, Searles R et al. Loss of the nuclear receptor corepressor SLIRP compromises male fertility. PLOS ONE 2013; 8: e70700.

49 Piao YR, Jin ZH. Loss of Gufl impairs sperm mitochondrial function and leads to male infertility. Biol Reprod 2015; 92: 5.

50 Holtman CK, Pawlyk AC, Meadow ND, Pettigrew DW. Reverse genetics of Escherichia coli glycerol kinase allosteric regulation and glucose control of glycerol utilization in vivo. $J$ Bacteriol 2001; 183: 3336-3344.

51 Ellerman DA, Brantua VS, Martinez SP, Cohen DJ, Conesa D, Cuasnicu PS. Potential contraceptive use of epididymal proteins: immunization of male rats with epididymal protein DE inhibits sperm fusion ability. Biol Reprod 1998; 59: 1029-1036.

52 Ye X, Chun J. Lysophosphatidic acid (LPA) signaling in vertebrate reproduction. Trends Endocrinol Metab 2010; 21: 17-24.

53 Qiu Q, Liu G, Li W, Shi Q, Zhu F, Lu G. Glycerol-3phosphate acyltransferase 4 gene is involved in mouse spermatogenesis. Acta Biochim Biophys Sinica 2009; 41: 668-676.

54 Ohba Y, Sakuragi T, Kage-Nakadai E et al. Mitochondriatype GPAT is required for mitochondrial fusion. EMBO J 2013; 32: 1265-1279.

55 Lambert MW. Functional significance of nuclear alpha spectrin. J Cell Biochem 2015; 116: 1816-1830.

(Supplementary information is linked to the online version of the paper on the Cell Discovery website.)

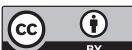

This work is licensed under a Creative Commons Attribution 4.0 International License. The images or other third party material in this article are included in the article's Creative Commons license, unless indicated otherwise in the credit line; if the material is not included under the Creative Commons license, users will need to obtain permission from the license holder to reproduce the material. To view a copy of this license, visit http://creativecommons.org/licenses/by/4.0/

(C) The Author(s) 2017 